\title{
Semaphorin4D Induces Inhibitory Synapse Formation by Rapid Stabilization of Presynaptic Boutons via MET Coactivation
}

@Cátia P. Frias, Jian Liang, Tom Bresser, Lisa Scheefhals, Matthijs van Kesteren, René van Dorland, Hai Yin Hu, Anna Bodzeta, Paul M.P. van Bergen en Henegouwen, Casper C. Hoogenraad, and $₫$ Corette J. Wierenga

Cell Biology, Department of Biology, Science4Life, Utrecht University, 3584 CH Utrecht, The Netherlands

Changes in inhibitory connections are essential for experience-dependent circuit adaptations. Defects in inhibitory synapses are linked to neurodevelopmental disorders, but the molecular processes underlying inhibitory synapse formation are not well understood. Here we use high-resolution two-photon microscopy in organotypic hippocampal slices from GAD65-GFP mice of both sexes to examine the signaling pathways induced by the postsynaptic signaling molecule Semaphorin4D (Sema4D) during inhibitory synapse formation. By monitoring changes in individual GFP-labeled presynaptic boutons, we found that the primary action of Sema4D is to induce stabilization of presynaptic boutons within tens of minutes. Stabilized boutons rapidly recruited synaptic vesicles, followed by accumulation of postsynaptic gephyrin and were functional after $24 \mathrm{~h}$, as determined by electrophysiology and immunohistochemistry. Inhibitory boutons are only sensitive to Sema4D at a specific stage during synapse formation and sensitivity to Sema4D is regulated by network activity. We further examined the intracellular signaling cascade triggered by Sema4D and found that bouton stabilization occurs through rapid remodeling of the actin cytoskeleton. This could be mimicked by the actin-depolymerizing drug latrunculin B or by reducing ROCK activity. We discovered that the intracellular signaling cascade requires activation of the receptor tyrosine kinase MET, which is a well known autism risk factor. By using a viral approach to reduce MET levels specifically in inhibitory neurons, we found that their axons are no longer sensitive to Sema4D signaling. Together, our data yield important insights into the molecular pathway underlying activitydependent Sema4D-induced synapse formation and reveal a novel role for presynaptic MET at inhibitory synapses.

Key words: inhibitory synapses; live imaging; organotypic slices; synapse formation; two-photon microscopy

Significance Statement

GABAergic synapses provide the main inhibitory control of neuronal activity in the brain. We wanted to unravel the sequence of molecular events that take place when formation of inhibitory synapses is triggered by a specific signaling molecule, Sema4D. We find that this signaling pathway depends on network activity and involves specific remodeling of the intracellular actin cytoskeleton. We also reveal a previously unknown role for MET at inhibitory synapses. Our study provides novel insights into the dynamic process of inhibitory synapse formation. As defects in GABAergic synapses have been implied in many brain disorders, and mutations in MET are strong risk factors for autism, our findings urge for a further investigation of the role of MET at inhibitory synapses.

\section{Introduction}

GABAergic synapses provide the main inhibitory control over neuronal activity in the brain and are indispensable for shaping network function (Isaacson and Scanziani, 2011). Although the majority of synaptic connections are formed during development, synapse turnover is still ongoing in postnatal brain tissue to 
allow for continuous adaptation and learning (Caroni et al., 2012). Recent studies have indicated that activity-dependent formation and disassembly of inhibitory synapses are crucial in experience-dependent circuit adaptation (Hensch, 2005; Keck et al., 2011; Chen et al., 2015; Froemke, 2015; Sprekeler, 2017), and defects in GABAergic synapses have been observed in many neurodevelopmental disorders (Marín, 2012; Cellot and Cherubini, 2014; Nelson and Valakh, 2015). We and others have shown that inhibitory synapses are dynamic structures with presynaptic boutons and postsynaptic scaffolds forming and disappearing with apparently stochastic dynamics (Wierenga et al., 2008; Fu and Huang, 2010; Dobie and Craig, 2011; Kuriu et al., 2012; Schuemann et al., 2013; Villa et al., 2016). These dynamics allow quick adaptation of connections in response to changes in the neuronal circuitry (Staras, 2007; Keck et al., 2011; Frias and Wierenga, 2013; Chen et al., 2015).

Previous work has shown that the formation of inhibitory synapses is a highly dynamic process, which starts with the formation of a new bouton by the presynaptic axon and takes several hours, up to 1-2 d, to be complete (Wierenga et al., 2008; Dobie and Craig, 2011; Schuemann et al., 2013; Flores et al., 2015). In recent years, enormous progress has been made by the identification and characterization of proteins that are involved in the formation of inhibitory synapses (Siddiqui and Craig, 2011; Krueger-Burg et al., 2017; Lu et al., 2017), but the precise molecular events that take place when new synapses are formed are not yet clear. For instance, boutons do not emerge randomly, but appear and reappear at specific axonal locations (Staras, 2007; Dobie and Craig, 2011; Schuemann et al., 2013), which seem to be predefined by a currently unknown mechanism. Furthermore, only some boutons get stabilized at these locations to form mature synapses (Wierenga et al., 2008; Fu et al., 2012; Schuemann et al., 2013; Villa et al., 2016) and it is currently not clear what determines the decision to "stay or go".

The class 4 semaphorin Sema4D, originally identified as an axon guidance factor (Kolodkin et al., 1993; Pasterkamp, 2012), was shown to be specifically required for the formation of GABAergic, but not glutamatergic, synapses in primary cultures as well as in vivo (Paradis et al., 2007). It was further shown that activation of the Sema4D signaling pathway rapidly induces the formation of inhibitory synapses (Kuzirian et al., 2013). Sema4D is a postsynaptic membrane protein and induces inhibitory synapse formation via presynaptic PlexinB1 receptors (Raissi et al., 2013; McDermott et al., 2018), but the precise molecular events that take place during Sema4D-induced inhibitory synapse formation are not known. The observation that somatic and dendritic inhibitory synapses respond equally to Sema4D signaling (Kuzirian et al., 2013) suggests that it acts at the majority of (or perhaps all) inhibitory synapses, making Sema4D signaling an interesting starting point to study the process of inhibitory synapse formation.

We used high-resolution two-photon microscopy in organotypic hippocampal slices to characterize the molecular events during Sema4D-induced formation of inhibitory synapses in intact tissue. We found that Sema4D signaling does not induce the formation of de novo synapses, but specifically promotes the rapid stabilization of inhibitory boutons along the axon in an activity-dependent manner. Rapid presynaptic changes are followed by subsequent slower recruitment of postsynaptic gephyrin, and maturation to functional inhibitory synapses completes over the course of several hours. The intracellular pathway for bouton stabilization involves specific remodeling of the actin cytoskeleton. We demonstrate that Sema4D-induced inhibitory bouton stabilization requires the activation of the receptor tyrosine kinase MET. Our data unravel an important regulatory pathway of activity-dependent inhibitory synapse formation and reveal a novel role for presynaptic MET in Sema4D-induced formation of inhibitory synapses.

\section{Materials and Methods}

Animals. All animal experiments were performed in compliance with the guidelines for the welfare of experimental animals issued by the Federal Government of The Netherlands. All animal experiments were approved by the Animal Ethical Review Committee (DEC) of Utrecht University.

Hippocampal slice cultures. Hippocampal slice cultures ( $400 \mu \mathrm{m}$ thick) were prepared from postnatal days 5-7 male and female GAD65-GFP mice (López-Bendito et al., 2004) as previously described (Müllner et al., 2015). In short, the hippocampi were dissected in ice-cold HEPES-GBSS [containing the following (in mM): $1.5 \mathrm{CaCl}_{2}, 0.2 \mathrm{KH}_{2} \mathrm{PO}_{4}, 0.3 \mathrm{MgSO}_{4}, 5$ $\mathrm{KCl}, 1 \mathrm{MgCl}_{2}, 137 \mathrm{NaCl}, 0.85 \mathrm{Na}_{2} \mathrm{HPO}_{4}$, and $\left.12.5 \mathrm{HEPES}\right]$ supplemented with $1 \mathrm{~mm}$ kynurenic acid and $25 \mathrm{~mm}$ glucose, and plated in a MEMbased medium (MEM supplemented with 25\% HBSS, 25\% horse serum, $30 \mathrm{~mm}$ glucose, and $12.5 \mathrm{~mm}$ HEPES).

In GAD65-GFP mice, $\sim 20 \%$ of the CA1 interneurons express GFP from early embryonic developmental stage into adulthood (LópezBendito et al., 2004; Wierenga et al., 2010). The majority of GFP-labeled interneurons expresses reelin and VIP, whereas parvalbumin and somatostatin expression is nearly absent (Wierenga et al., 2010). For our study, the relatively low number of GFP-positive axons is crucial for the proper analysis of individual boutons.

The slices were kept in culture for at least 1 week before the experiments [range $7-29$ days in vitro (DIV)] at $35^{\circ} \mathrm{C}$ in $5 \% \mathrm{CO}_{2}$. For live imaging experiments, slices were transferred to an imaging chamber, where they were continuously perfused with carbogenated artificial CSF [ACSF; containing the following (in mM): $126 \mathrm{NaCl}, 3 \mathrm{KCl}, 2.5 \mathrm{CaCl}_{2}, 1.3$ $\mathrm{MgCl}_{2}, 1.25 \mathrm{NaH}_{2} \mathrm{PO}_{4}, 26 \mathrm{NaHCO}_{3}, 20$ glucose, and 1 Trolox). The temperature of the chamber was maintained at $35-37^{\circ} \mathrm{C}$. Treatment and control experiments were conducted in slices from the same culture.

Pharmacological treatments. The following pharmacological treatments were used: 0.1/0.2\% DMSO, $1 \mathrm{~nm} \mathrm{Fc}$, and Sema4D-Fc (amino acids 24-711; both R\&D Systems), 100 nм latrunculin B (Santa Cruz Biotechnology), $200 \mathrm{~nm}$ Jasplakinolide (Tocris Bioscience), $1 \mu \mathrm{M}$ PHA665752 (Sigma-Aldrich), $10 \mu \mathrm{M}$ Y-27632 (Sigma-Aldrich), tetrodotoxin citrate (TTX; Tocris Bioscience), $20 \mu \mathrm{M}$ 6,7-dinitroquinoxaline-2,3dione disodium salt (DNQX; Tocris Bioscience) and $50 \mu \mathrm{M}$ DL-2-amino5-phosphonopentanoic acid (APV; Tocris Bioscience). We used the small molecule PHA-665752 (PHA), a highly specific MET inhibitor (Christensen et al., 2003), to decrease endogenous phosphorylation of MET without affecting MET expression or neuronal cell viability (Christensen et al., 2003; Lim and Walikonis, 2008)

For treatments that were followed by immunostaining of inhibitory synapses, 1 nм Fc or Sema4D-Fc was added to the culturing medium and slices were left in the incubator for 2, 6, or $24 \mathrm{~h}$ before fixation.

Two-photon imaging. Time-lapse two-photon microscopy images were acquired on a Femtonics $2 \mathrm{D}$ two-photon laser-scanning microscope, with a Nikon CFI Apochromat $60 \times$ NIR water-immersion objective. GFP was excited using a laser beam tuned to $910 \mathrm{~nm}$ (Mai Tai HP, Spectra Physics). The 3D images $(93.5 \times 93.5 \mu \mathrm{m}$ in $x, y ; 1124 \times 1124$ pixels $)$ consisted of $29-33 z$-stacks $(0.5 \mu \mathrm{m}$ step size in $z)$. Small misalignments because of drift were manually corrected during the acquisition.

For acute treatments, drugs were added to the perfusion ACSF after a baseline period of $40 \mathrm{~min}$ [ 5 time points (TPs)] and we continued imaging for an additional 10 TPs in the wash-in period (total imaging period is $140 \mathrm{~min}$ ). In longer treatments, we treated the slices for $6 \mathrm{~h}$ after the baseline period ( 5 imaging TPs) at the microscope and restarted imaging for five TPs, for a total treatment period of $6 \mathrm{~h}$ and $40 \mathrm{~min}$ (400 $\mathrm{min}$ ). Morphology of the axons did not change during this period, indicating slice health was well preserved. For activity blockade, $0.5 \mu \mathrm{M}$ TTX was added to the perfusion ACSF before the transfer of the slice to the imaging chamber. 
Two-photon image analysis. The analysis of inhibitory bouton dynamics was performed semiautomatically using ImageJ (U.S. National Institutes of Health) and MATLAB-based software (MathWorks). The 3D coordinates of individual axons were selected at every time point using the CellCounter plugin (Kurt De Vos, University of Sheffield, Academic Neurology, Sheffield, UK). For each image, $1-5$ axons (average length 78 $\mu \mathrm{m}$ with SD $18 \mu \mathrm{m}$, with average of 31 boutons per axon with SD 10; for local treatment experiments, average length $39 \mu \mathrm{m}$ with SD $8 \mu \mathrm{m}$, with average of 14 boutons per axon with SD of 4) were selected for analysis.

A 3D intensity profile along the selected axons was constructed at each time point, and individual boutons were identified in a two-step process using custom-made MATLAB software (Schuemann et al., 2013). In brief, an axon threshold was calculated to differentiate the axon from the background (2 SD above mean intensity); subsequently, a local threshold (0.5 SD above mean axon intensity) identified the boutons along the selected axon. Only boutons with at least five pixels above local threshold were included. Each image stack was visually examined, and falsepositives and false-negatives were corrected manually. Only raw data were analyzed; images were median-filtered for illustration purposes only.

Boutons were classified as persistent when they were present during all time points, and non-persistent when they were absent during one or more time points during the imaging session. Per axon, we calculated the average fraction of persistent and non-persistent boutons by normalization to the average number of boutons for each time point. To bias our analysis toward synaptic events (Schuemann et al., 2013), we restricted our analysis to boutons that appeared for at least two time points at the same location during the imaging period. We verified that our main conclusions did not change when this restriction was released. Based on their presence during baseline and treatment periods, we defined five subgroups of non-persistent boutons: new boutons (not present during baseline), lost boutons (not present during wash-in), stabilizing boutons (non-persistent during baseline, persistent during wash-in), destabilizing boutons (persistent during baseline, non-persistent during wash-in), and intermittent boutons (non-persistent in baseline and wash-in; Fig. 1). The average fraction of each subgroup of boutons was normalized to the total average number of non-persistent boutons per axon. Bouton density was calculated as the average number of boutons at all time points divided by the $3 \mathrm{D}$ axon length.

Local treatment experiments. For the local treatment experiments, we used HEPES-ACSF [containing the following (in mM): $126 \mathrm{NaCl}, 3 \mathrm{KCl}$, $2.5 \mathrm{CaCl}_{2}, 1.3 \mathrm{MgCl}_{2}, 1.25 \mathrm{NaH}_{2} \mathrm{PO}_{4}, 20$ glucose, and 10 HEPES, pH 7.4] with $20 \mu \mathrm{M}$ AlexaFluor 568 (Invitrogen), to visualize the spread of the local application. Sema4D or Fc was added to the HEPES-ACSF to a final concentration of $10 \mathrm{~nm}$. We increased the concentration compared with bath application experiments to account for fast dilution. The solution was loaded into a patch pipette $(4-6 \mathrm{M} \Omega)$, and was locally applied to a GFP-labeled axon using a Picospritzer II (General Valve). Time-lapse two-photon microscopy imaging was performed as described previously, except that a second laser (Mai Tai HP, Spectra Physics) was used at 840 $\mathrm{nm}$ to visualize the area of the puff and images were taken at every $5 \mathrm{~min}$. The $3 \mathrm{D}$ images $(51.3 \times 51.3 \mu \mathrm{m}$ in $x, y ; 620 \times 620$ pixels $)$ consisted of $18-22 z$-stacks $(0.5 \mu \mathrm{m}$ step size in $z)$. After a baseline period of $20 \mathrm{~min}(5$ TPs), the pipette was put into position before the stimulation. The stimulation consisted of 300 puffs of $20-50 \mathrm{~ms}$ at $2 \mathrm{~Hz}$. The pipette was carefully retracted before continuing the time series for 10 additional TPs (total imaging period of $70 \mathrm{~min}$ ). Images were analyzed as described in the previous section.

Electrophysiology. During the experiment, the slice was placed in a recording chamber perfused with carbogenated ACSF at a rate of $1 \mathrm{ml} /$ $\mathrm{min}$. The recording ACSF consisted of the following (in $\mathrm{mM}$ ): $126 \mathrm{NaCl}, 3$ $\mathrm{KCl}, 2.5 \mathrm{CaCl}_{2}, 1.3 \mathrm{MgCl}_{2}, 1.25 \mathrm{Na}_{2} \mathrm{H}_{2} \mathrm{PO}_{4}, 26 \mathrm{NaHCO}_{3}$, and 20 glucose. Whole-cell voltage-clamp recordings were performed at $35^{\circ} \mathrm{C}$ in $\mathrm{CA} 1$ cells of GAD65-GFP slice cultures at 13-19 DIV. Recordings were made on a MultiClamp 700B amplifier (Molecular Devices) and stored using pClamp 10 software. To isolate sIPSCs, $20 \mu \mathrm{M}$ DNQX and $50 \mu \mathrm{M}$ APV were added to the recording ACSF. For mIPSCs, $0.5 \mu \mathrm{M}$ TTX was added as well. Thick-walled borosilicate pipettes of 3-6 $\mathrm{M} \Omega$ were filled with an internal solution containing the following (in $\mathrm{mm}$ ): $70 \mathrm{~K}$-gluconate, 70
KCl, 0.5 EGTA, 10 HEPES, 4 MgATP, $0.4 \mathrm{NaGTP}$, and $4 \mathrm{Na}_{2}$ phosphocreatine. Cells were excluded from analysis if the series resistance increased $>35 \%$ over the course of the experiment. IPSCs were automatically detected in Clampfit and further analyzed in custom MATLAB scripts. Detected events within $3 \mathrm{~ms}$ of each other were merged and events smaller than three times the RMS of the signal were excluded.

Immunohistochemistry, confocal imaging, and image analysis. For post hoc immunohistochemistry, an auto-fluorescent laser "scar" was made after live imaging by performing a line scan at high intensity to mark the imaged region. Organotypic hippocampal slices were fixed in $4 \%(\mathrm{w} / \mathrm{v})$ paraformaldehyde (PFA) for $30 \mathrm{~min}$ at room temperature. Slices were rinsed in phosphate buffer (PBS) and permeabilized with $0.5 \%$ Triton $\mathrm{X}-100$ in PBS for $15 \mathrm{~min}$. Slices were then blocked with $0.2 \%$ Triton X-100 and 10\% normal goat serum (ab7481, Abcam) in PBS for 60 min. Primary antibodies were applied overnight at $4^{\circ} \mathrm{C}$ in blocking solution. After washing, slices were incubated with secondary antibodies in blocking solution for $4 \mathrm{~h}$ at room temperature. Slices were washed and mounted on slides in Vectashield mounting medium (Vector Laboratories).

The following primary and secondary antibodies were used: rabbit $\alpha$-VGAT (1:1000; Synaptic Systems, 131 003; RRID:AB_887869), mouse $\alpha$-gephyrin (1:1000; Synaptic Systems, 147 011; RRID:AB_887717), guinea pig $\alpha$-VGLUT1 (1:400; Millipore, AB5905; RRID:AB_2301751), rabbit $\alpha$-Homer1 (1:1000; Synaptic Systems, 160 002; RRID: AB_2120990), mouse $\alpha$-myc (1:100; Oncogene Research Products, OP10), mouse $\alpha$-MET (1:500; Santa Cruz Biotechnology, sc-8057; RRID: AB_673755), AlexaFluor 405-, AlexaFluor 488-, and AlexaFluor 568conjugated secondary antibodies (Invitrogen). For staining MET, we also used a previously described myc-tagged nanobody, which was shown to recognize MET with low nanomolar affinity (Heukers et al., 2014). We visualized the nanobody with an antibody against the C-terminal myc tag. We validated the MET staining in primary hippocampal cultures using a previously described immunostaining protocol (Esteves da Silva et al., 2015).

For immunostainings, high resolution confocal laser scanning microscopy was performed on a Zeiss LSM-700 system with a Plan-Apochromat $63 \times 1.4$ NA oil-immersion objective. Each image was a $z$-series of 11-35 images $(0.3 \mu \mathrm{m} z$-step size $)$, each averaged four times. The imaging area in the CA1 region was $78 \times 78 \mu \mathrm{m}(1024 \times 1024$ pixels $)$. Settings were kept the same to compare fluorescence intensities between slices.

To account for within-slice variability, we took confocal images from separate regions (maximally 5 per slice) as independent measurements. For the quantification of vesicular GABA transporter (VGAT) and gephyrin intensities per image, we determined the mean intensity of 3 randomly chosen areas of $10 \times 10 \mu \mathrm{m}$ of the average projection image from the five middle $z$-stacks. For the cumulative plots individual values (per area) were used. Synaptic puncta size and number of the same image areas were determined using the PunctaAnalyzer plugin, and inhibitory synapses were defined as overlapping VGAT and gephyrin puncta.

For determining colocalization of GFP-labeled boutons with synaptic markers VGAT or gephyrin, or with MET, we manually inspected individual boutons through all $z$-sections. A bouton was only considered positive when at least one $z$-stack of the bouton overlapped with VGAT, gephyrin or MET staining. Images were median-filtered for illustration purposes only. To check for random overlap between MET puncta and GFP boutons, colocalization was determined for images in which the MET channel was inverted.

STED imaging. Dual-color STED imaging was performed in primary neurons with a Leica TCS SP8 STED $3 \times$ microscope using a HC PL APO $100 \times / 1.4$ oil-immersion STED WHITE objective. Primary hippocampal neurons were prepared as previously described (Esteves da Silva et al., 2015). Neurons (18 DIV) were fixed with $4 \%$ PFA and $4 \%$ sucrose in PBS for $10 \mathrm{~min}$ at room temperature and washed three times with PBS supplemented with $100 \mathrm{~mm}$ glycine (PBS/Gly). Next, cells were permeabilized and blocked with $100 \mathrm{~mm}$ glycine, $0.1 \%$ Triton-X, and 10\% normal goat serum in $\mathrm{PBS}$ for $1 \mathrm{~h}$ at $37^{\circ} \mathrm{C}$. Neurons were then incubated for $2-3$ $\mathrm{h}$ at room temperature with primary antibodies diluted in PBS supplemented with $100 \mathrm{~mm}$ glycine, $0.1 \%$ Triton-X, and 5\% normal goat serum. Next, cells were washed three times with PBS/Gly and incubated for $1 \mathrm{~h}$ at 
A Persistent $(\mathrm{P} ; \triangleright)$

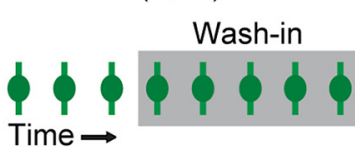

Time $\rightarrow$

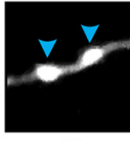

0

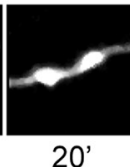

20

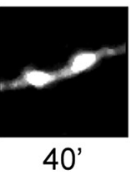

$40^{\prime}$

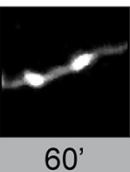

$60^{\prime}$

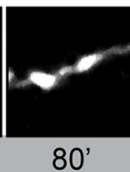

$80^{\prime}$

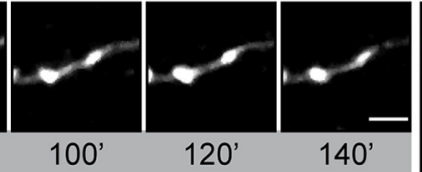

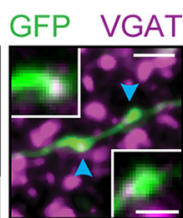

B Non-persistent (NP; present - $>$ - or absent - $\gg$-)

1. New $(\mathrm{N})$

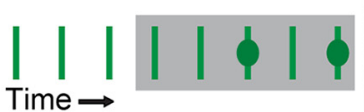

2. Lost (L)

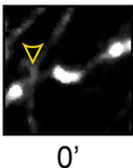

$0^{\prime}$
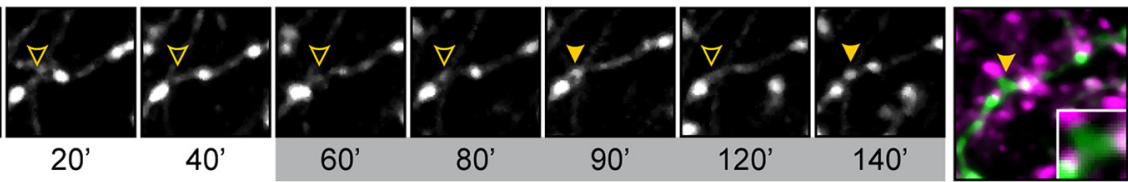

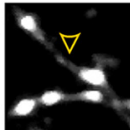

0

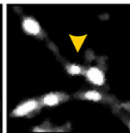

20

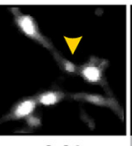

30 '
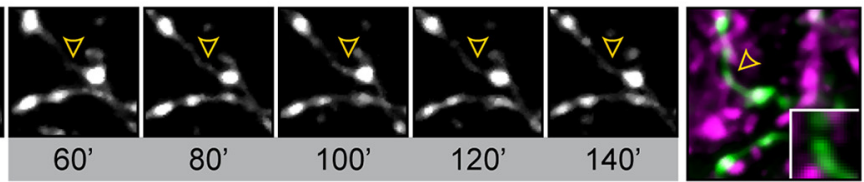

3. Stabilizing (S)
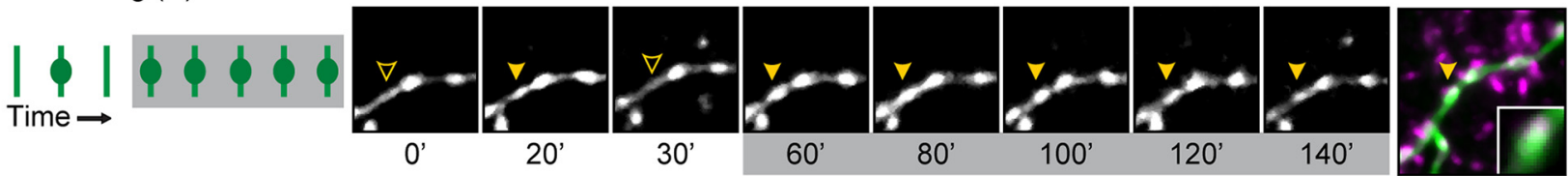

4. Destabilizing (D)
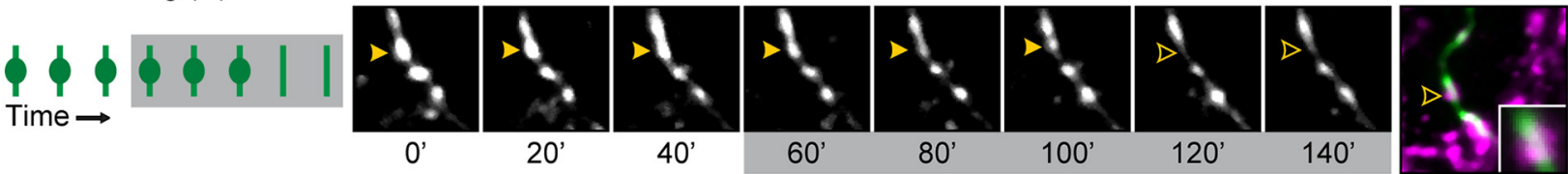

5. Intermittent (I)
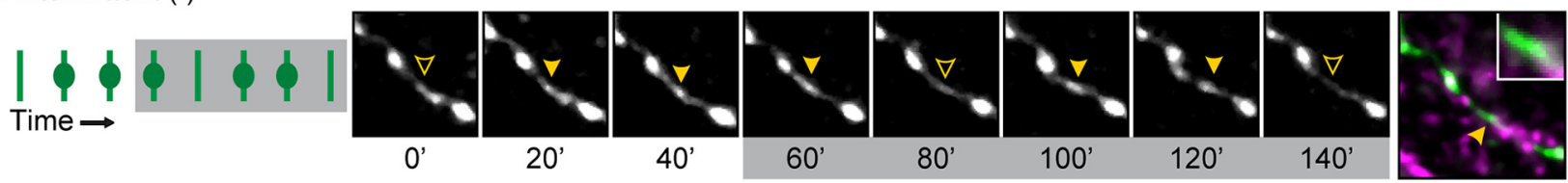

C

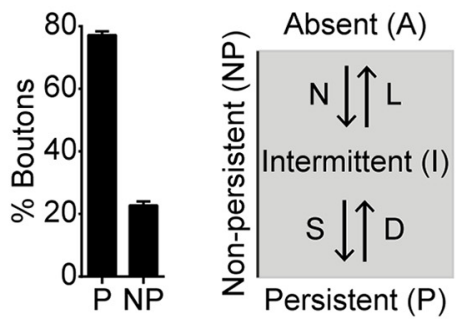

E

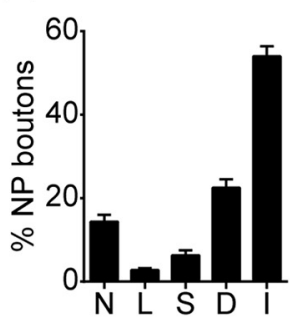

$\mathbf{F}$

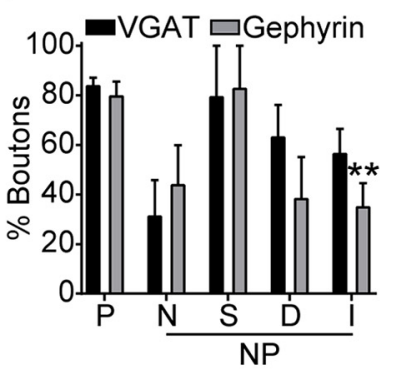

G

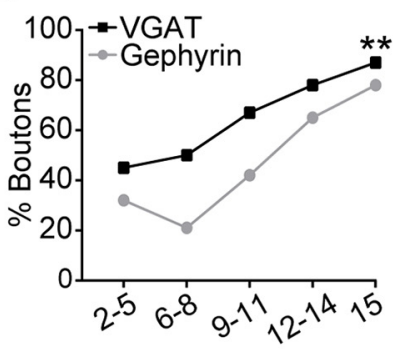

Bouton lifetime (TPs)

Figure 1. Classification of presynaptic inhibitory boutons by their dynamics. A, Time-lapse two-photon images of two inhibitory boutons (blue arrowheads) along a GAD65-GFP-labeled axon in the CA1 region of the hippocampus. These boutons were present at all time points, and were therefore categorized as persistent (P) boutons. Only every second image is shown for clarity. On the right, the same region is shown after fixation and staining against vesicular GABA transporter (VGAT; magenta). The zoom shows a single optical plane through the bouton to demonstrate overlap (white) of VGAT and GFP boutons (green). Time in minutes. Scale bars: $2 \mu \mathrm{m}$; inset, $1 \mu \mathrm{m}$. $\boldsymbol{B}$ 1-B5, Same as in $\boldsymbol{A}$, showing examples of non-persistent (NP boutons: new (B1; absent during baseline), lost (B2; absent during wash-in), stabilizing ( $\boldsymbol{B} 3$; NP during baseline, $\mathrm{P}$ during wash-in), destabilizing ( $\boldsymbol{B 4}$; P during baseline, NP during wash-in), and intermittent (B5; NP during both baseline and wash-in) boutons. Filled yellow arrowheads indicate that the bouton is present, and empty yellow arrowheads indicate that the bouton is absent at the time point shown. $C$, Average fraction of $P$ and NP boutons at any given time point. $D$, Schematic illustration of bouton classification. We consider three developmental stages of inhibitory boutons at axon-dendrite crossings: absent (A), NP or $P$. Within the group of NP boutons, we define five subgroups, reflecting transitions between these stages: $N:$ new $(A \rightarrow N P), L:$ lost $(N P \rightarrow A), S: \operatorname{stabilizing}(N P \rightarrow P), D:$ destabilizing $(P \rightarrow N P)$, and I: intermittent (always NP) boutons. $E$, Average fraction of the five subgroups of NP boutons normalized to the total number of NP boutons. $F$, Fraction of boutons positive for VGAT and gephyrin per axon. Two-way ANOVA showed a significant effect on bouton type $(p=0.008)$. For gephyrin, Pversus I, ${ }^{* *} p=0.001$ (Sidak's multiple-comparisons test). G, Fraction of boutons colocalizing with VGAT or gephyrin as a function of bouton lifetime (total number of time points (TPs) present during the imaging period). L boutons were not included. $\chi^{2}:$ TP2-TP5, $p=0.36 ;$ TP6 -TP8, $p=0.11$; TP9-TP11, $p=0.15 ;$ TP12-TP14, $p=0.33$ : TP15, ${ }^{* *} p=0.008$. Confocal images in $\boldsymbol{A}$ and $\boldsymbol{B}$ are maximum intensity projections of 5-8 $z$-stacks, whereas two-photon images are maximum intensity projections of 13-15z-stacks. Data are represented as mean \pm SEM. Data in $\boldsymbol{C}$ and $\boldsymbol{E}$ are from 90 axons from 24 independent experiments; data in $\boldsymbol{F}$ from 21 axons from five independent experiments for the VGAT staining (P:n=282 boutons; $\mathrm{N}: n=14 ; \mathrm{S}: n=6 ; \mathrm{D}: n=17 ; 1: n=44$ ) and from 15 axons from 4 independent experiments for the gephyrin staining (P:n=232 boutons; $\mathrm{N}: n=15 ; \mathrm{S}: n=6 ; \mathrm{D}: n=15 ; \mathrm{I}: n=39)$. In G, $n=14-29$ per TP, except for TP15. 
room temperature with secondary antibodies diluted in PBS supplemented with $100 \mathrm{~mm}$ glycine, $0.1 \%$ Triton-X, and $5 \%$ normal goat serum. Cells were then washed three times with PBS/Gly and mounted in Mowiol mounting medium. Abberior STAR 580 was excited with $561 \mathrm{~nm}$ and Abberior STAR 635P with $633 \mathrm{~nm}$ pulsed laser light $(80 \mathrm{MHz})$. Both Abberior STAR 580 and 635P were depleted with a $775 \mathrm{~nm}$ pulsed depletion laser. Fluorescence emission was detected using Leica HyD hybrid detector.

The following primary and secondary antibodies were used: rabbit $\alpha$-VGAT (1:1000; Synaptic Systems, 131 003; RRID:AB_887869), mouse $\alpha$-MET (1:500; Santa Cruz Biotechnology, sc-8057; RRID:AB_673755), Abberior STAR 635P $\alpha$-mouse (1:200; Abberior, 2-0002-007-5) and Abberior STAR $580 \alpha$-rabbit (1:200; Abberior, 2-0012-005-8).

miRNA and DNA constructs. The miRNA sequences targeting the ORF of mouse full-length MET cDNA (NM_008591) were designed with the BLOCK-iT RNAi Designer (Sequence 1: 5' -GCAGTGAATTAGTTCGC TATG-3'; Sequence 2: 5'-GCTTGTTGACACATACTATGA-3'). Sequence 1 was based on a previously published shRNA (Qiu et al., 2014). A scrambled sequence was generated with the siRNA Sequence Scrambler from Genscript (Sequence: 5'-ACTATAGTAATGCTCGTGCAT$\left.3^{\prime}\right)$. A loop sequence was added (5'-GTTTTGGCCACTGACTGAC-3') and oligos were annealed and cloned into pSM155-GFP (Du et al., 2006). The miRNA cassette was then cloned into a lentiviral plasmid (FUGW; RRID:Addgene_14883), which also contains GFP. A double-floxed (lox2272/loxp) inverse orientation version of these plasmids was created for Cre-dependent expression of the miRNA and GFP.

cDNA for overexpression of MET was obtained from pBabe puro MET WT (RRID:Addgene_17493). A PCR was performed with forward primer 5'-AAGGCGCGCCGCCACCATGAAGGCTCCCACCGT-3' and reverse primer 5'-AAGGATCCATGTGTTCCCCTCGCC-3'. This fragment was ligated into a pGW2-mCherry vector resulting in a C-terminal tagged MET.

Verification of knock down. Endogenous MET levels were too low to detect reliably with Western blot and we therefore relied on MET overexpression to verify our miRNA constructs. Primary hippocampal neurons were transfected using Lipofectamine 2000 (Invitrogen) at 9 DIV. Briefly, MET-mCherry and miRNA or GFP only constructs ( total 2.1 $\mu \mathrm{g} /$ well, for a 12 -well plate) were mixed with $3.3 \mu \mathrm{l}$ Lipofectamine 2000 in $100 \mu \mathrm{l}$ Neurobasal medium (NB), incubated for $25 \mathrm{~min}$, and then added to the neurons in $\mathrm{NB}$ with $0.5 \mathrm{~mm}$ glutamine at $37^{\circ} \mathrm{C}$ in $5 \% \mathrm{CO}_{2}$ for $40 \mathrm{~min}$. Next, neurons were washed in preheated NB and transferred back to their original medium for $48 \mathrm{~h}$. Neurons were fixed with $4 \%$ PFA + $4 \%$ sucrose for $10 \mathrm{~min}$ and mounted on microscope slides with Vectashield mounting medium (Vector Laboratories). Images were acquired using a Zeiss LSM-700 confocal laser scanning microscope. The imaging settings were kept the same between experiments to compare fluorescence intensity in the soma.

HEK293 cells (American Type Culture Collection, CRL-1573; RRID: CVCL_0045) were grown in $10 \mathrm{~cm}$ dishes at $37^{\circ} \mathrm{C}$ and $5 \% \mathrm{CO}_{2}$, and transfected with MET-mCherry and miRNA or GFP only constructs using polyethylenimine (PEI; $1 \mu \mathrm{g} / \mu \mathrm{l}$; Polysciences) to assess MET levels by Western blot analysis. After 24-48 h, HEK293 cells were lysed in Laemmli sample buffer (10\% glycerol, 2\% SDS, 100 mM DTT, 50 mM Tris- $\mathrm{HCl} \mathrm{pH} 6.8$ and $0.004 \%$ bromophenol blue) and scrapped, and samples were then boiled for $10 \mathrm{~min}$. Samples were loaded equally and run on a $10 \%$ SDS-PAGE gel. Proteins were transferred to Nitrocellulose membranes (Bio-Rad, 1620115) and blocked in 2\% (w/v) bovine serum albumin in PBS/0.1\% (v/v) Tween 20 for $1 \mathrm{~h}$ at room temperature. Primary antibodies were diluted in the blocking solution and applied overnight at $4^{\circ} \mathrm{C}$. Membranes were washed three times in PBS/0.1\% Tween 20 and incubated with secondary IRDye antibodies for $1 \mathrm{~h}$ at room temperature. Afterward, membranes were washed three times in PBS/0.1\% Tween 20, and scanned using an Odyssey Infrared Imaging System (LICOR Biosciences). MET intensity was corrected for tubulin loading control, and values for miRNAs were normalized to GFP control values.

The following primary and secondary antibodies were used: mouse $\alpha$-MET (1:250; Santa Cruz Biotechnology, sc-8057; RRID:AB_673755), rabbit $\alpha$-tubulin (1:10,000; Abcam, ab52866; RRID:AB_869989), IRdye 680LT $\alpha$-mouse (1:20,000; LI-COR Biosciences, 926-68020; RRID:
AB_10706161), and IRdye 800CW $\alpha$-rabbit (1:15,000; LI-COR Biosciences, 926-32211; RRID:AB_621843).

Lentivirus production and infection. HEK293 cells were grown in DMEM supplemented with $10 \%$ FBS and $1 \%$ penicillin/streptomycin (Pen/Strep) at $37^{\circ} \mathrm{C}$ and $5 \% \mathrm{CO}_{2}$. A confluent $10 \mathrm{~cm}$ dish of HEK293 cells was split 1:4 the day before transfection. Per $10 \mathrm{~cm}$ dish the following mixture was used for transfection: $2 \mu \mathrm{g}$ pMD2.G (RRID: Addgene_12259), $4 \mu \mathrm{g}$ psPAX2 (RRID:Addgene_12260), $6 \mu \mathrm{g}$ FUGW (RRID:Addgene_14883), $500 \mu \mathrm{l}$ OptiMEM, and $36 \mu \mathrm{l}$ PEI $(1 \mu \mathrm{g} / \mu \mathrm{l}$; Polysciences). After $15 \mathrm{~min}$ incubation at room temperature, the mixture was added to the cells. Culture medium was replaced with $5 \mathrm{ml} \mathrm{Op-}$ tiMEM supplemented with $1 \%$ Pen/Strep 4-6h later. Lentivirus containing supernatant was harvested after 48-60 h. Virus were concentrated with Amicon spin filters (Millipore), aliquoted and stored at $-80^{\circ} \mathrm{C}$.

Cre-dependent expression of miRNA (Sequence 2) and GFP was induced in organotypic slices of VGAT-Cre mice (RRID:IMSR_JAX: 028862). Stereotactic injections of Lentiviral constructs (miRNA Sequence $2+$ GFP) were performed in 1 DIV slices using a microinjector (Eppendorf, FemtoJet) and stereoscopic microscope (Leica, M80). Per slice, $6-8$ injections were done in the CA1 stratum radiatum with $\sim 50 \mathrm{~Pa}$ pressure ( $<1 \mu \mathrm{l}$ virus solution per slice). For control, we used a lentiviral construct for Cre-dependent GFP expression without miRNA sequence. Imaging and analysis of GFP-labeled axons in VGAT-Cre slices was performed at 14-29 DIV as described in the sections Two-photon imaging and Two-photon image analysis.

Experimental design and statistical analysis. All data analysis was performed blind to the treatment. Live imaging experiments were performed in pairs (control + treatment) per batch of slices, and for each pair of experiments, slices from different animals were used. Data are represented as mean values \pm SEM, unless stated otherwise. Statistical analysis was performed using GraphPad Prism software. Results from treatment and control experiments were compared using the MannWhitney $U$ test (MW). The $\chi^{2}$ test was used for comparing the fraction of axons with/without stabilizing boutons and the fraction of boutons with synaptic markers VGAT and gephyrin. For comparing multiple groups, we used the Kruskal-Wallis test (KW) followed by a post hoc Dunn's comparison test. We used a one-way ANOVA followed by a Dunnett's multiple-comparison test (one-way ANOVA) to compare the effect of wash-in of PHA over time during the electrophysiological recordings. Treatment effects at multiple time points were compared using two-way ANOVA followed by a Sidak's multiple-comparisons test (two-way ANOVA). For the comparison of cumulative distributions, we used the Kolmogorov-Smirnov (KS) test. We have indicated the tests and $p$ values in the figure legends. Differences between control and treatment were considered significant when $p<0.05{ }^{*} p<0.05,{ }^{* *} p<0.01,{ }^{* * *} p<$ $0.001)$. In all figure legends and text, $N$ indicates the number of independent experiments (slices or neuronal cultures), and $n$ indicates the number of axons/images analyzed.

\section{Results}

We performed time-lapse two-photon microscopy in organotypic hippocampal cultures from GAD65-GFP mice to monitor the dynamics of inhibitory boutons in the CAl region of the hippocampus (Wierenga et al., 2008; Schuemann et al., 2013). In GAD65-GFP mice, the majority of GFP-labeled interneurons are dendritically targeting CGE-derived interneurons, whereas MGE-derived parvalbumin and somatostatin interneurons are not labeled (López-Bendito et al., 2004; Wierenga et al., 2010). This results in GFP-labeling of $\sim 20 \%$ of CA1 interneurons, allowing monitoring of individual axons over time. Highresolution image stacks of GFP-labeled inhibitory axons were acquired every $10 \mathrm{~min}$, for a total period of $140 \mathrm{~min}$ [15 time points (TPs)].

As previously reported (Wierenga et al., 2008; Dobie and Craig, 2011; Fu et al., 2012; Schuemann et al., 2013), inhibitory boutons are remarkably dynamic and many boutons appeared, disappeared and reappeared during the course of the imaging 
period. To bias our analysis toward synaptic events, we only included boutons that were present for at least two TPs at the same location during the imaging period (Schuemann et al., 2013). We distinguished two main classes of boutons: persistent boutons, which were present during all time points (Fig. 1A), and nonpersistent boutons, which were absent during one or more time points during the imaging session (Fig. 1B). In our slices, the majority of GFP-labeled boutons (77\%, with SD of $12 \%)$ were persistent (Fig. 1C) and they reflect mature inhibitory synapses (Wierenga et al., 2008; Müllner et al., 2015). Non-persistent boutons reflect locations where inhibitory synapses are "in transition", e.g., where synapses are being formed or disassembled in an apparent trial-and-error fashion (Wierenga et al., 2008; Dobie and Craig, 2011; Fu et al., 2012; Schuemann et al., 2013).

We assume that synapse formation is reflected in subsequent transitions of boutons from absent (A) to non-persistent (NP) to persistent $(\mathrm{P})(\mathrm{A} \rightarrow \mathrm{NP} \rightarrow \mathrm{P})$ and synapse disassembly in the reverse order. We identify these transitions by comparing the dynamics of individual boutons in a baseline and wash-in period and we define five subgroups of NP boutons: new $(\mathrm{A} \rightarrow \mathrm{NP})$, lost $(\mathrm{NP} \rightarrow \mathrm{A})$, stabilizing $(\mathrm{NP} \rightarrow \mathrm{P})$, destabilizing $(\mathrm{P} \rightarrow \mathrm{NP})$, and intermittent (always NP) boutons (Fig. $1 B, D$; for details, see Materials and Methods). We could observe transitions between developmental bouton stages in $\sim 50 \%$ of NP boutons (Fig. $1 E$ ). After live imaging, slices were fixed and immunostained for the presynaptic VGAT and the postsynaptic scaffold gephyrin to correlate bouton dynamics of individual boutons with their molecular composition. We found that boutons that were NP at the end of the imaging period (new, destabilizing, and intermittent boutons) showed a lower percentage of association with VGAT and gephyrin compared with $\mathrm{P}$ boutons, reflecting that these boutons usually do not (yet) form synapses (Fig. $1 F$ ). Stabilizing boutons, which had been present for at least $90 \mathrm{~min}$ before fixation, showed similar association with VGAT and gephyrin to P boutons (Fig. $1 F$ ), indicating that they represent nascent inhibitory synapses that have started to recruit presynaptic and postsynaptic proteins within this period. Association of NP boutons with VGAT and gephyrin increased with their total lifetime, suggesting a gradual recruitment of proteins over the imaging period (Fig. 1G). Recruitment of gephyrin was delayed compared with VGAT, consistent with earlier reports that synaptic proteins are recruited in a presynaptic before postsynaptic order (Wierenga et al., 2008; Dobie and Craig, 2011). These data demonstrate that inhibitory presynaptic boutons are continuously being formed and disassembled along axons. By monitoring the dynamics of individual inhibitory boutons over time we can distinguish boutons at different stages of synapse assembly and disassembly.

\section{Sema4D treatment induces stabilization of inhibitory boutons}

We used our live imaging method to examine whether Sema4D signaling affects a specific step in the process of inhibitory synapse formation. We bath applied the extracellular domain of mouse Sema4D-conjugated to the Fc region of mouse IgG2A (Sema4D; $1 \mathrm{nM}$ ) and compared inhibitory bouton dynamics during a baseline period of five TPs and during Sema4D treatment in the subsequent 10 TPs (Fig. 2A). We used Fc (1 nM) as a control treatment (Kuzirian et al., 2013). Bath application of Sema4D did not affect axonal morphology (Fig. $2 A$ ) and did not change the overall density of inhibitory boutons (Fig. $2 B$ ). However, when we analyzed the different subgroups of NP boutons, we found that Sema4D treatment resulted in an enhanced fraction of stabilizing boutons from $6 \pm 2$ to $16 \pm 3 \%$ (Fig. $2 C$ ). The absolute density of stabilizing boutons was increased by $>2$-fold, while the other subgroups of boutons were unaffected by Sema4D (Fig. $2 D-H)$. Noticeably, new boutons were unaffected (Fig. $2 D$ ), indicating that Sema4D treatment did not induce de novo bouton formation. To examine how bouton stabilization developed over time, we quantified the number of boutons that were present for five consecutive TPs during the baseline and the wash-in period. Sema4D induced a marked increase in bouton stabilization over the course of the wash-in period (Fig. 2I), and strongly increased the number of boutons that were stabilized by the end of the imaging period (Fig. 2J). Stabilizing boutons were relatively rare in our slices, as under control conditions most axons display no, or at most one, stabilizing bouton (Fig. $2 K$ ). Treatment with Sema4D significantly increased the fraction of axons with one or more stabilizing boutons (Fig. $2 \mathrm{~K}$ ). Longer Sema4D treatment (6 h) did not increase bouton stabilization beyond the $2 \mathrm{~h}$ level (Fig. $2 L$ ), indicating that only a limited number of inhibitory boutons can be stabilized by Sema4D. Altogether, these data show that Sema4D treatment in intact tissue does not induce de novo bouton formation, but rather specifically promotes the stabilization of inhibitory presynaptic boutons, without affecting synapse elimination.

\section{Sema4D-induced stabilization of inhibitory boutons is the first step of inhibitory synapse formation}

We wanted to verify that Sema4D-induced inhibitory bouton stabilization leads to the formation of functional synapses in our slices. We treated organotypic hippocampal slices with $1 \mathrm{nM} \mathrm{Fc}$ or $1 \mathrm{~nm}$ Sema4D for $24 \mathrm{~h}$, and recorded miniature inhibitory postsynaptic currents (mIPSCs; Fig. 3A). Treatment with Sema4D increased the mIPSC frequency by $37 \%$ (from $5.2 \pm 0.5$ to $7.1 \pm$ $0.5 \mathrm{~Hz}$ ), whereas mIPSC amplitude was not affected (Fig. $3 \mathrm{~B}, \mathrm{C}$ ). We also determined overall inhibitory synapse density by colocalizations of presynaptic VGAT and postsynaptic gephyrin (Fig. $3 D, E)$. Consistent with the electrophysiology results, Sema4D induced a clear $24 \pm 7 \%$ increase in the density of inhibitory synapses (Fig. $3 F$ ). These results demonstrate that the bouton stabilization observed within $2 \mathrm{~h}$ after Sema4D treatment leads to the formation of new functional synapses after $24 \mathrm{~h}$.

A previous study has shown that Sema4D can induce rapid changes in postsynaptic gephyrin in primary neurons (Kuzirian et al., 2013), but it was not addressed whether these postsynaptic changes preceded or followed presynaptic changes. To determine the time course of the recruitment of presynaptic and postsynaptic elements during Sema4D-induced synapse formation, we quantified VGAT and gephyrin immunostaining after 2, 6, and $24 \mathrm{~h}$ treatments. Treatment with Sema4D induced an increase in the area of VGAT puncta, without affecting their density (Fig. $3 G-I)$, consistent with an effect on stabilization, and not de novo formation, of inhibitory boutons. For gephyrin, Sema4D treatment caused an increase in puncta density, but not in their size (Fig. 3J-L), suggesting that stabilizing boutons were recruiting gephyrin. The average puncta intensity was not affected [at $24 \mathrm{~h}$, VGAT: $107 \pm 4 \%$ of control, $p=0.35$ (MW); gephyrin: $106 \pm$ $5 \%$ of control, $p=0.51(\mathrm{MW})]$. Interestingly, the time course for presynaptic and postsynaptic changes was different. Whereas an increase in presynaptic VGAT area could already be detected after $6 \mathrm{~h}$ (Fig. $3 G$ ), the increase in postsynaptic gephyrin density was only evident after $24 \mathrm{~h}$ (Fig. $3 K$ ). This suggests that Sema4D signaling induced a gradual increase in presynaptic vesicle content in stabilized boutons and subsequent acquisition of postsynaptic scaffolds to form new inhibitory synapses. Our data indicate 
A
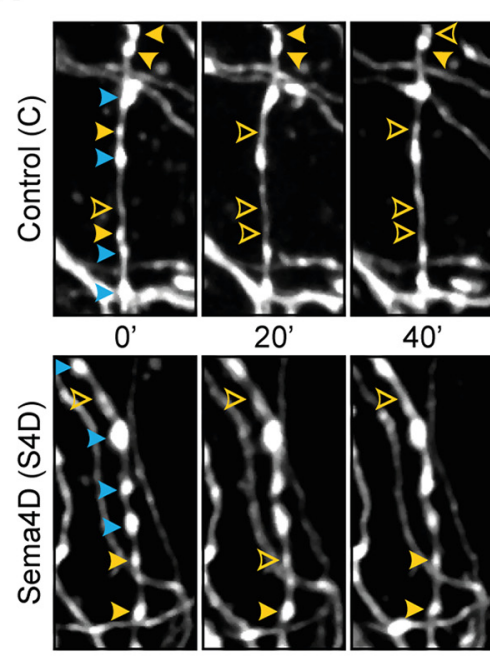

$>$ Persistent bouton

$>$ Non-persistent bouton absent/present

40

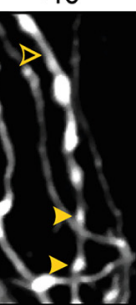

$+\mathrm{C}$
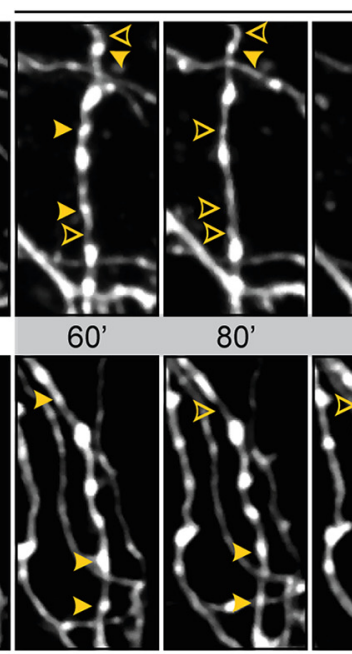

$80^{\prime}$
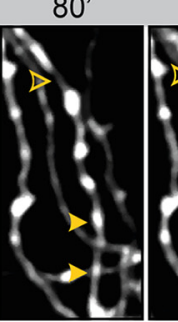

$100^{\prime}$
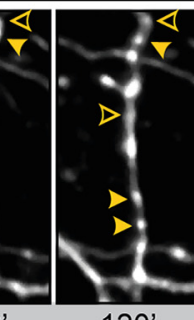

$120^{\prime}$

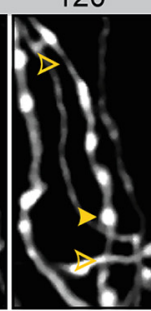

$+S 4 \mathrm{D}$
B

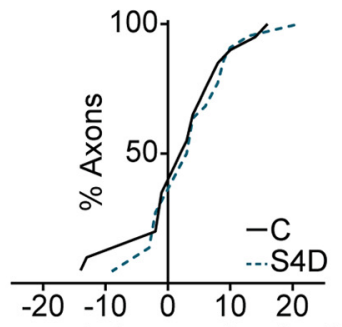

Change in bouton density (\%)

C

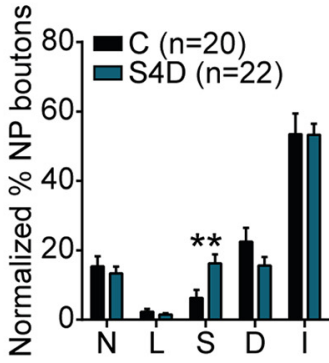

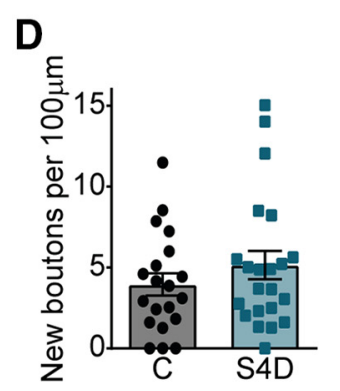
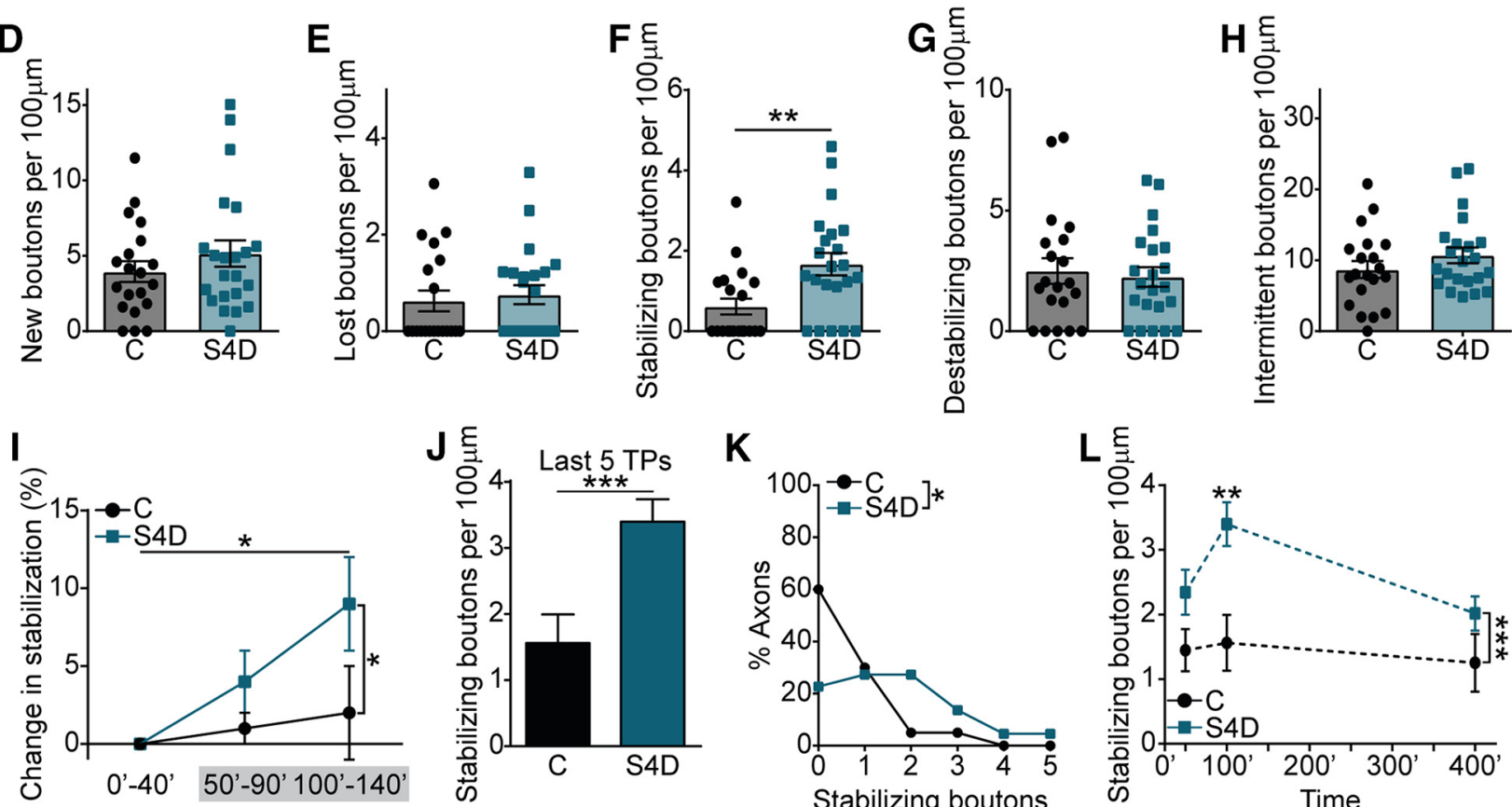

K

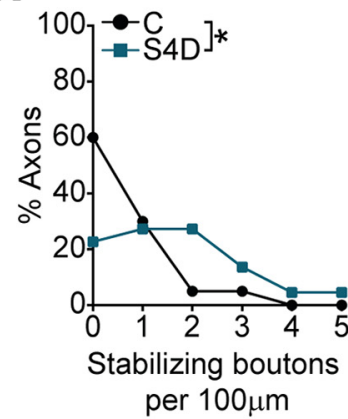

L

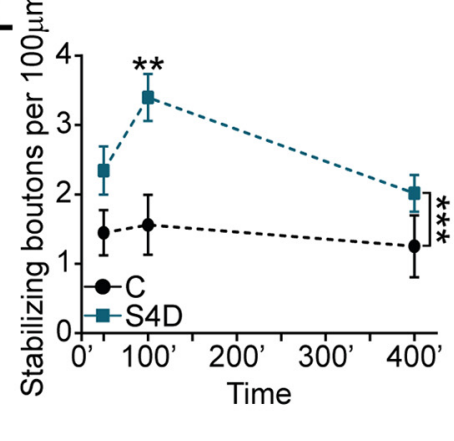

Figure 2. Sema4D treatment promotes inhibitory bouton stabilization. $\boldsymbol{A}$, Time-lapse two-photon images of GFP-labeled inhibitory axons in the CA1 region of the hippocampus during baseline [5 time points (TPS)] and wash-in (10 TPs; gray box) of $1 \mathrm{~nm} \mathrm{Fc}$ - control (C; top) or $1 \mathrm{~nm} \mathrm{Sema4D-Fc} \mathrm{(S4D;} \mathrm{bottom).} \mathrm{Only} \mathrm{every} \mathrm{second} \mathrm{image} \mathrm{is} \mathrm{shown} \mathrm{for} \mathrm{clarity.} \mathrm{P} \mathrm{(blue)} \mathrm{and} \mathrm{NP} \mathrm{(yellow)} \mathrm{boutons} \mathrm{are}$ indicated by arrowheads. Filled arrowheads indicate that the bouton is present, and empty arrowheads indicate that the bouton is absent at that time point. Images are maximum intensity projections of 11-18z-stacks. Time in minutes. Scale bar, $5 \mu \mathrm{m}$. B, Cumulative distribution of the change in mean bouton density during the wash-in period compared with baseline after wash-in of C or S4D (MW, $p=0.83)$. C, Average fraction of subgroups of NP boutons in C- and S4D-treated axons: N: new (MW, $p=0.86)$; L: lost (MW, $p=0.93) ; S:$ stabilizing (MW, $\left.{ }^{* *} p=0.003\right) ; \mathrm{D}$ : destabilizing (MW, $p=0.25)$; I: intermittent (MW, $p=0.89)$. $\boldsymbol{D}-\boldsymbol{H}$, Density of new (D;MW, $p=0.41)$, lost $(\boldsymbol{E} ; \mathrm{MW}, p=0.61)$, stabilizing $\left(\boldsymbol{F} ; \mathrm{MW}\right.$, $\left.{ }^{* *} p=0.003\right)$, destabilizing $(\mathbf{G} ; \mathrm{MW}, p=0.84)$,

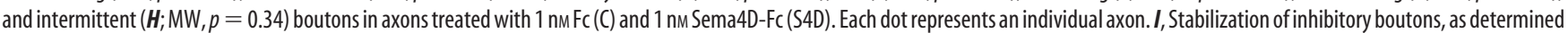
by the change (compared with baseline) in density of boutons that were present at five consecutive TPs during the imaging period: $0^{\prime}-40^{\prime}$ (baseline), $50^{\prime}-90^{\prime}$ (wash-in, gray box), and 100' $-140^{\prime}$ (wash-in, gray box). Two-way ANOVA showed a significant effect of both treatment ( ${ }^{*} p=0.04$ ) and time ( ${ }^{*} p=0.03$ ). J, Density of boutons that stabilized in the last five TPs (MW, ${ }^{* * *} p=0.0008$ ). $\boldsymbol{K}$, Frequency distribution of the stabilizing bouton density in C- and S4D-treated axons $\left(\chi^{2},{ }^{*} p=0.03\right)$. $L$, Density of stabilizing boutons after treatment with $C$ or $S 4 D$ for 50,100 , and 400 min. Two-way ANOVA showed that S4D increased bouton density independent of time $\left.{ }^{* * *} p=0.0002\right)$. At $100^{\prime},{ }^{* *} p=0.005$ (Sidak's multiple-comparisons test). Data are represented as mean \pm SEM. Data from 20 control axons $(N=6)$ and 22 S4D-treated axons $(N=5)$. Data in $L$ at $400^{\prime}$ from 15 control axons $(N=4)$ and 17 S4D-treated axons $(N=4)$.

that Sema4D signaling primarily acts via the presynaptic axon and that the changes in postsynaptic gephyrin are secondary.

The previously reported rapid increase in postsynaptic gephyrin clusters after Sema4D treatment in primary cultures
(Kuzirian et al., 2013) may be well explained by an overall difference in neuronal maturation level compared with our experiments. In young neurons, new gephyrin clusters can be rapidly induced by local GABA signaling (Oh et al., 2016), whereas in 
A

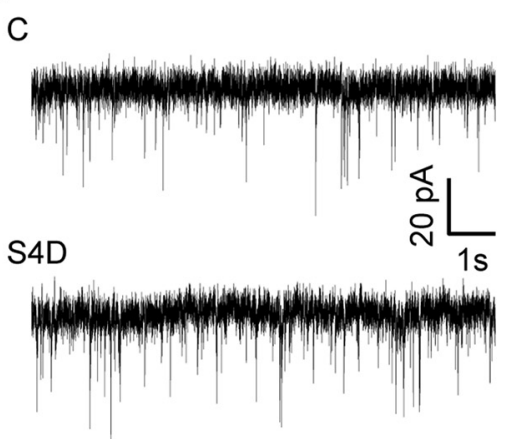

D

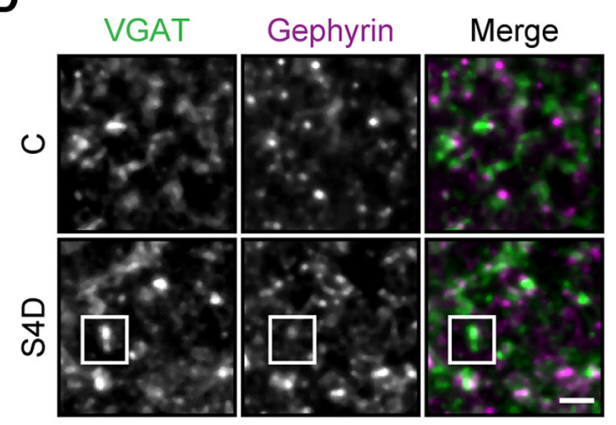

B

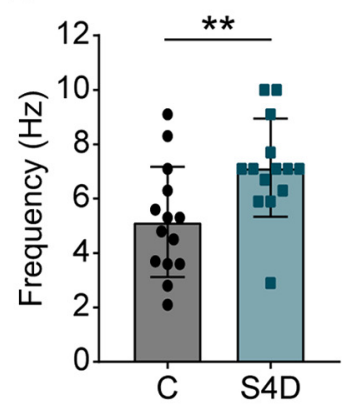

E

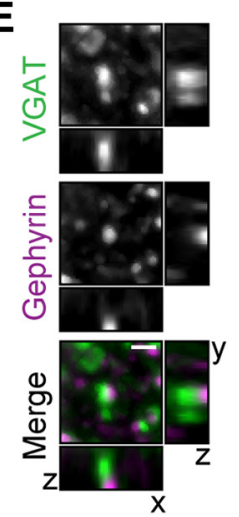

C

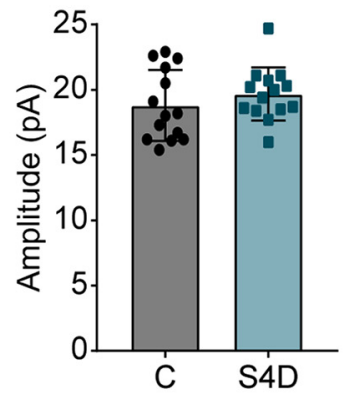

F

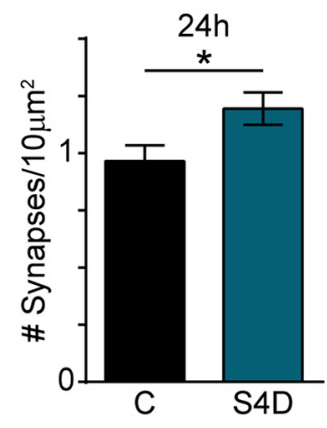

G

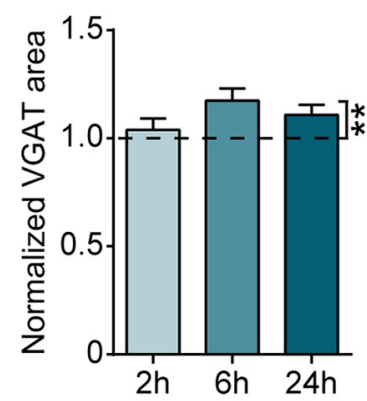

J

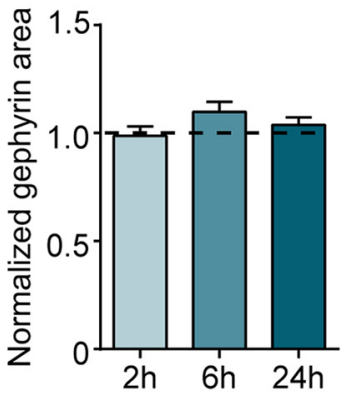

H

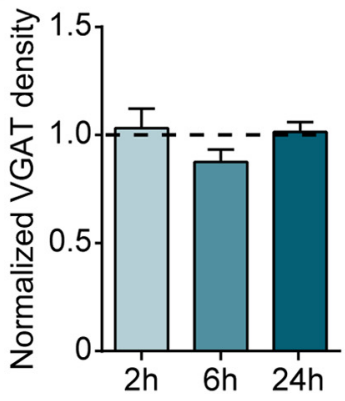

I

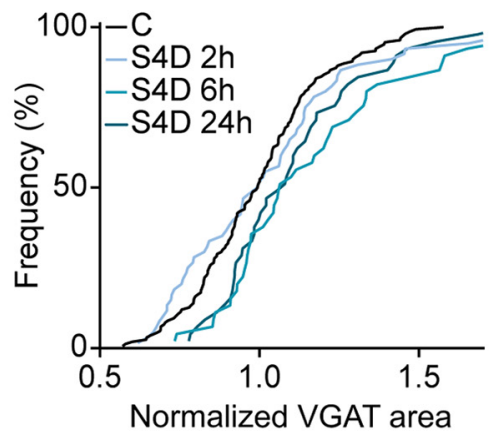

L

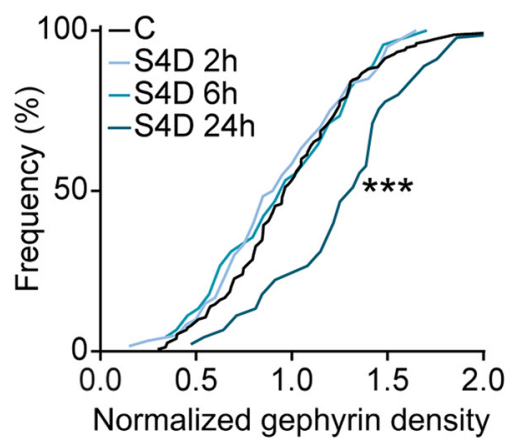

Figure 3. Sema $4 \mathrm{D}$ increases overall inhibitory synaptic density. $A$, Representative whole-cell voltage-clamp recordings of mIPSCs from CA1 pyramidal cells in organotypic hippocampal slices treated for $24 \mathrm{~h}$ with $1 \mathrm{~nm} \mathrm{Fc} \mathrm{(C)} \mathrm{or} 1 \mathrm{~nm} \mathrm{S4D} \mathrm{(S4D).} \mathrm{B,} \mathrm{Mean} \mathrm{mIPSC} \mathrm{frequency} \mathrm{after} 24 \mathrm{~h}$ treatment with Cor S4D (MW, ${ }^{* *} p=0.008$ ). C, Mean mIPSC amplitude after $24 \mathrm{~h}$ treatment with C or S4D

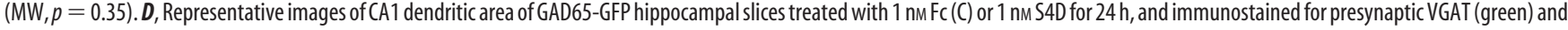
gephyrin (magenta). Images are average intensity projections of five z-stacks. Scale bar, $2 \mu \mathrm{m}$. $\boldsymbol{E}$, Example of an inhibitory synapse ( $\boldsymbol{D}$, white box) identified as the apposition of VGAT (green) and gephyrin (magenta) puncta. The respective $x, z$ and $y, z$ projections show the close apposition of the two markers. Images are maximum intensity projections of six $z$-stacks. Scale bar, $1 \mu \mathrm{m}$. $F$, Density of inhibitory synapses in slices treated with Fc or S4D for $24 \mathrm{~h}\left(\mathrm{MW},{ }^{*} p=0.03\right.$ ). G, Normalized area of VGAT puncta (after treatment with $1 \mathrm{~nm}$ S4D for 2, 6, and 24h). Two-way ANOVA showed that S4D treatment increased VGAT area independent of time $\left({ }^{* *} p=0.005\right)$. $\boldsymbol{H}$, Normalized density of VGAT puncta, after treatment with $1 \mathrm{~nm} \mathrm{S4D} \mathrm{for} 2,6$, and $24 \mathrm{~h}$. $\boldsymbol{I}$, Cumulative distributions of the normalized area of VGAT after treatment with $1 \mathrm{~nm} S 4 \mathrm{D}$ for 2, 6 , and $24 \mathrm{~h}$. Black line represents the normalized control values. $p=0.81, p=0.08$, and $p=0.14$ (KS) for 2, 6, and $24 \mathrm{~h}$, respectively. $\boldsymbol{J}, \boldsymbol{K}$, Same as $\boldsymbol{G}$ and $\boldsymbol{H}$, but for normalized area $(\boldsymbol{J})$ and density $(\boldsymbol{K})$ of postsynaptic gephyrin puncta. In $\boldsymbol{K}$, Two-way ANOVA showed a significant effect (Figure legend continues.) 
A

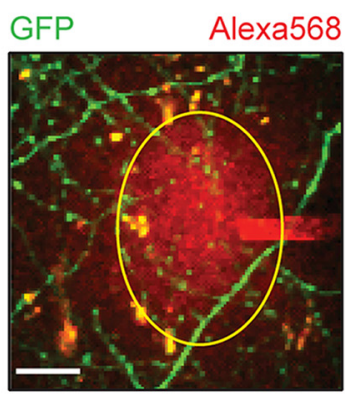

B

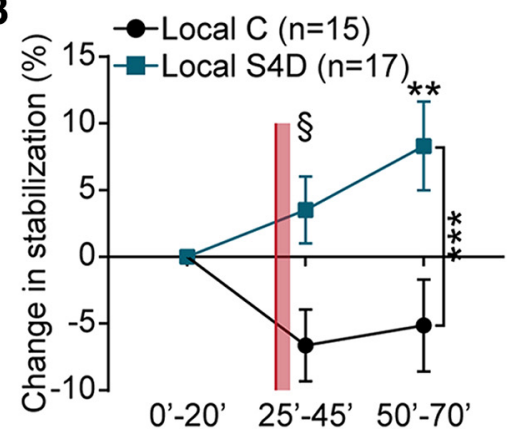

C

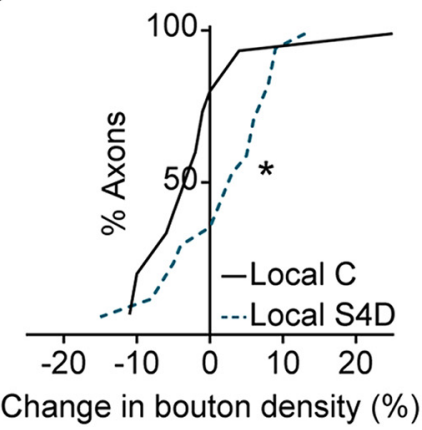

Figure 4. Sema4D induces local stabilization of inhibitory boutons. $\boldsymbol{A}$, Representative image of the local treatment of GFP-labeled inhibitory axons in the CA1 region of the hippocampus. AlexaFluor 568 (red) was added to the pipette to visualize the area of the puff (yellow circle). Scale bar, $10 \mu \mathrm{m}$. B, Stabilization of inhibitory boutons, as determined by the change (compared with

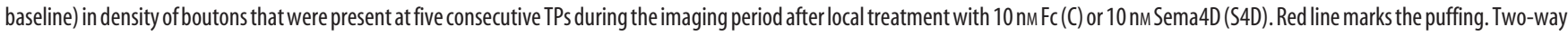
ANOVA showed a significant effect of treatment $\left({ }^{* * *} p=0.0002\right)$ and an interaction between treatment and time $(\$ p=0.02)$. At $50^{\prime}-70^{\prime},{ }^{* *} p=0.003($ Sidak's multiple-comparisons test). $\boldsymbol{C}$, Cumulative distribution of the change in mean bouton density after local treatment with C or S4D compared with baseline (MW, $\left.{ }^{*} p=0.045\right)$. Data are represented as mean \pm SEM. Data from 15 control axons $(N=6)$ and 17 S4D-treated axons $(N=6)$.

mature neurons prolonged or additional signaling appears to be required. In our slices, Sema4D treatment increased inhibitory synapse density by $\sim 25 \%$ after $24 \mathrm{~h}$ (Fig. $3 F$ ), which is comparable to experience-dependent changes in inhibitory synapses observed in vivo (Keck et al., 2011; Chen et al., 2015; Villa et al., 2016).

\section{Local application of Sema4D suggests competition between presynaptic boutons}

Under physiological conditions, Sema4D is a membraneattached protein acting locally at the synapse (Pasterkamp, 2012; Raissi et al., 2013), which is very different from the bath-applied Sema4D treatment that we use in our experiments. To examine possible differences between local and global Sema4D signaling, we locally applied Sema4D to short stretches ( $\sim 40 \mu \mathrm{m})$ of GFPlabeled inhibitory axons (Fig. 4A). Local application with control solution slightly reduced local bouton stabilization (compare Figs. $4 B, 2 I$, control curves), possibly because of mechanical pressure. In contrast, local application of Sema4D induced robust stabilization of inhibitory boutons in these axons (Fig. 4B). In fact, local application of Sema4D induced a significant increase in local bouton density (Fig. 4C), whereas bath application had failed to induce a change in overall bouton density (Fig. 2B). Presynaptic boutons are known to share presynaptic proteins and vesicles along the axon (Staras, 2007; Bury and Sabo, 2016). Our data suggest that stabilizing boutons compete for presynaptic components within individual axons when Sema4D is bath applied, limiting an increase in overall bouton density.

\section{Actin remodeling by low doses of latrunculin B promotes stabilization of inhibitory boutons}

Induction of inhibitory synapses in response to Sema4D was previously shown to be mediated by presynaptic PlexinB1 receptors (Kuzirian et al., 2013; McDermott et al., 2018). Bouton stabiliza-

\section{$\leftarrow$}

(Figure legend continued.) of time $(\$ p=0.04)$ and an interaction between treatment and time $(\$ p=0.04) . L$, Same as in $I$, but for normalized gephyrin density. $p=0.99, p=0.99$, and ${ }^{* * *} p<0.0001(\mathrm{KS})$ for 2,6 , and $24 \mathrm{~h}$, respectively. Data are represented as mean $\pm \mathrm{SEM}$. Data in $\boldsymbol{A}-\boldsymbol{C}$ are from 14 control cells $(\boldsymbol{N}=5)$ and 14 S4D-treated cells $(N=7)$; data in $\boldsymbol{F}$ from 15 control images $(N=3)$ and 15 S4D images $(N=3)$; and data in $\mathbf{G}-\boldsymbol{L}$ from 15 to 20 control images $(N=3-4)$ and 15-20S4D images $(N=3-4)$ per time point. Dashed lines in $\boldsymbol{G}, \boldsymbol{H}, \boldsymbol{J}$, and $\boldsymbol{K}$ represent control values (treatment with $1 \mathrm{~nm} F($ for 2, 6, and 24 h). Dots in $\boldsymbol{B}$ and $\boldsymbol{C r e p r e s e n t ~ i n d i v i d u a l ~ c e l l s . ~}$ tion is presumably induced by local changes in the intracellular actin cytoskeleton via Sema4D/PlexinB1 signaling pathways, which are well described in non-neuronal cells (Zhou et al., 2008; Cagnoni and Tamagnone, 2014). Interestingly, it was reported that Sema4D/PlexinB1 signaling can induce either polymerization or depolymerization of actin via RhoA regulation, depending on the coactivation of receptor tyrosine kinases MET and ErbB-2, which act as coreceptors for PlexinB1 (Swiercz et al., 2008; Sun et al., 2012). To examine how the actin cytoskeleton is involved in inhibitory bouton stabilization, we studied the effect of two actin remodeling drugs in our system with intended opposite effects: the actin monomer sequestering drug latrunculin $\mathrm{B}$ (LatB; $100 \mathrm{nM}$ ), which promotes actin depolymerization, and the actin stabilizer Jasplakinolide (Jasp; $200 \mathrm{~nm}$ ), which promotes actin polymerization. None of these actin drugs affected overall axon morphology (Fig. 5A). We analyzed bouton dynamics in the presence of these actin drugs and found that LatB treatment, and not Jasp treatment, increased the fraction of stabilizing boutons (Fig. $5 B, C$ ). The effect of LatB was highly specific for stabilizing boutons, as the other bouton subgroups were not affected (Fig. $5 C)$. Indeed, we found that LatB specifically increased the absolute density of stabilizing boutons by almost twofold (Fig. 5D) and increased the fraction of axons with stabilizing boutons (Fig. $5 E$ ), whereas Jasp did not change overall inhibitory bouton dynamics. The changes in bouton dynamics after LatB treatment were rapid and were surprisingly similar to Sema4D treatment (compare Figs. 5D,E, Fig. $2 F, K$ ). At these low concentrations, actin drugs do not affect synaptic function (Honkura et al., 2008; Rex et al., 2009), and we therefore assume that the observed effects reflect the direct action of the actin drugs on the local actin cytoskeleton. Our findings suggest that inhibitory bouton stabilization is promoted by conditions favoring local actin depolymerization, rather than polymerization.

The striking similarity between stabilization of inhibitory boutons induced by treatment with LatB or Sema4D suggests that both treatments induce a comparable effect on intracellular actin. Because we found that only a specific subset of inhibitory boutons was stabilized by Sema4D treatment, we tested whether these were the same boutons that responded to LatB. We treated slices with a combination of LatB and Fc, or LatB and Sema4D. We found that bouton stabilization by LatB occluded a further increase by co-application with Sema4D (Fig. 5 F, G). These results 
A
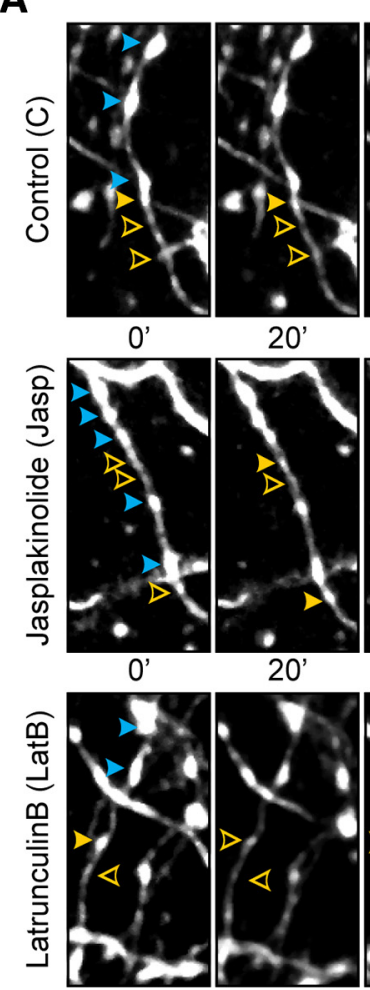

DE

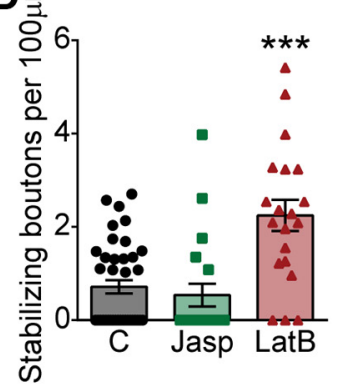

Wash-in

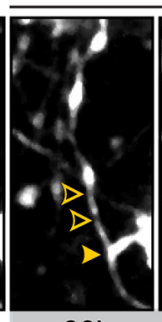

40
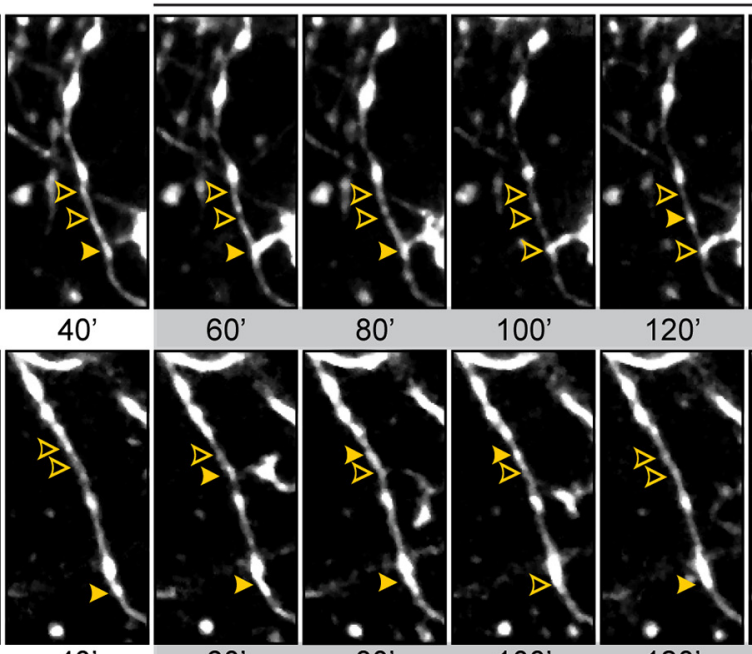

$40^{\prime}$
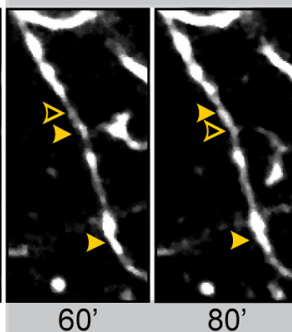

$100^{\prime}$
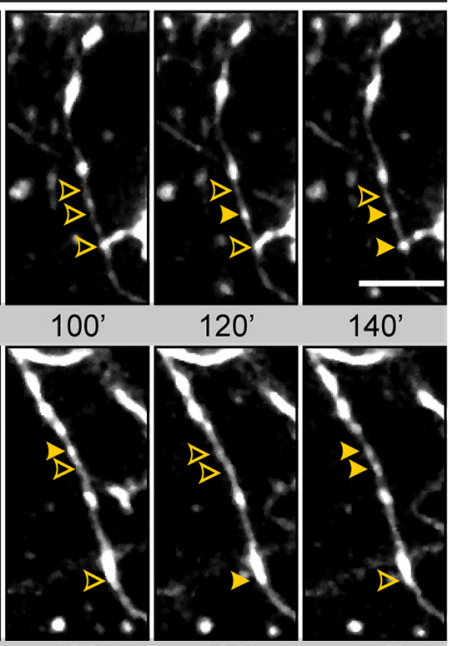
$>$ Persistent bouton
Non-persistent bouton
present
$>$ Non-persistent bouton
absent

B.

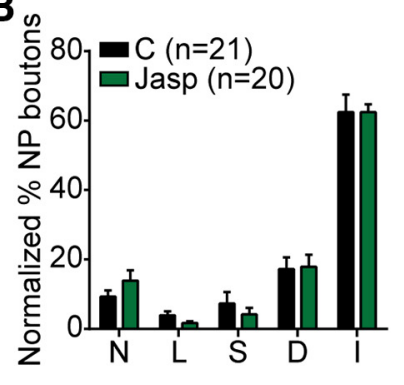

C
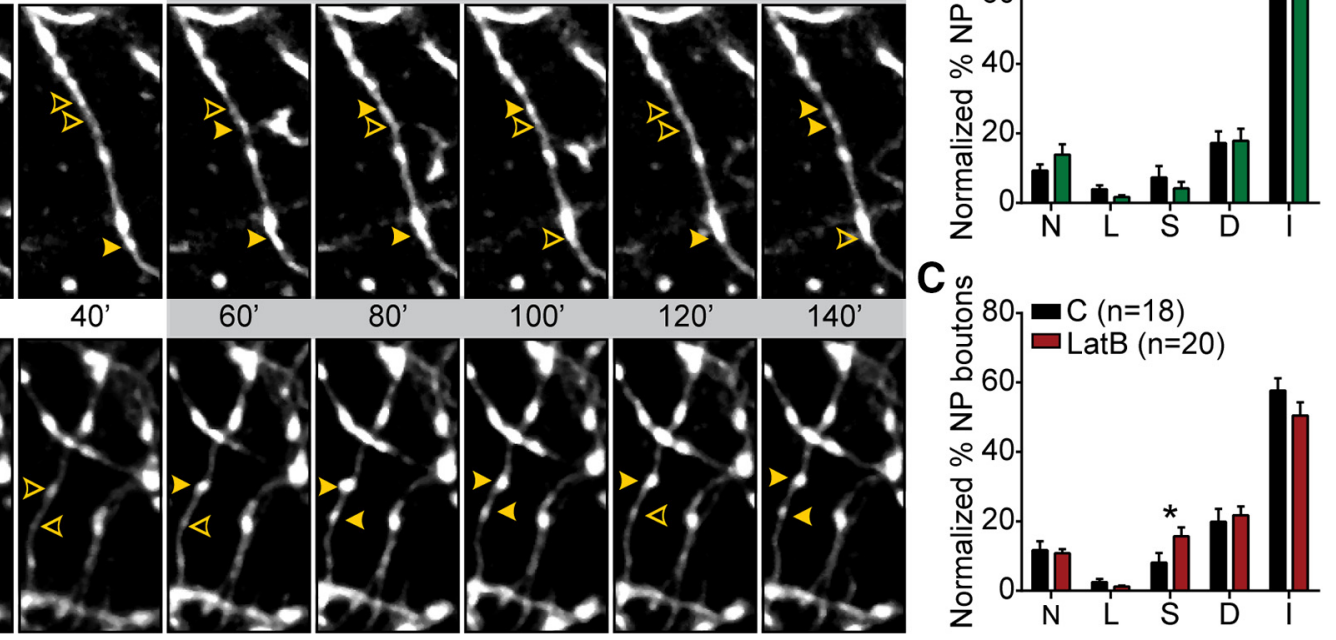

E

$\mathbf{F}$
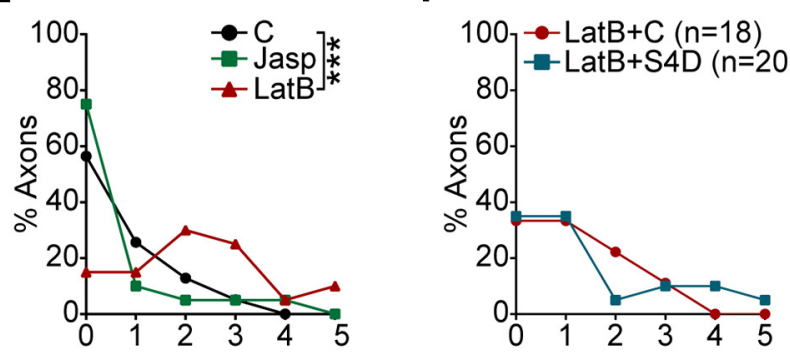

G

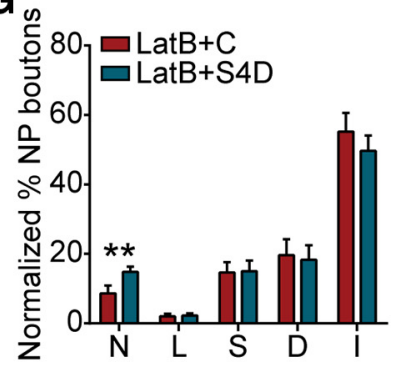

Stabilizing boutons per $100 \mu \mathrm{m}$ Stabilizing boutons per $100 \mu \mathrm{m}$

Figure 5. Inhibitory bouton dynamics are regulated by actin. $\boldsymbol{A}$, Time-lapse two-photon images of GAD65-GFP-labeled axons in the CA1 region of the hippocampus during baseline (5 TPs) and wash-in (10 TPs; gray box) of DMSO - control (C; top), $200 \mathrm{~nm}$ Jasplakinolide (Jasp; middle) or $100 \mathrm{~nm}$ LatrunculinB (LatB; bottom). Only every second image is shown for clarity. P and NP boutons are indicated as in Figure 2. Images are maximum intensity projections of 12-14 $z$-stacks. Time in minutes. Scale bar, $5 \mu \mathrm{m}$. $\boldsymbol{B}$, Fraction of NP boutons in C and Jasp-treated axons: $\mathrm{N}$ : new $(\mathrm{MW}, p=0.37)$; L: lost (MW, $p=0.18)$; S: stabilizing (MW, $p=0.49$ ); D: destabilizing (MW, $p=0.95$ ); I: intermittent (MW, $p=0.93)$. C, Same as in $\boldsymbol{B}$, but for C and LatB-treated axons (N: MW, $\left.p=0.99 ; \mathrm{L}: \mathrm{MW}, p=0.66 ; \mathrm{S}: \mathrm{MW},{ }^{*} p=0.01 ; \mathrm{D}: \mathrm{MW}, p=0.6 ; \mathrm{I}: \mathrm{MW}, p=0.29\right)$. D, Density of stabilizing boutons in C, Jasp- (MW, $\left.p=0.28\right)$, and LatB-treated axons (MW, $\left.{ }^{* * *} p<0.0001\right)$. Dots represent individual axons. $\boldsymbol{E}$, Frequency distribution of the stabilizing bouton density in $C$, Jasp-, and LatB-treated axons ( $\chi^{2} ;$ C vs Jasp, $p=0.31 ; C$ vs LatB, $\left.{ }^{* * *} p=0.0005\right)$. $\boldsymbol{F}$, Same as $\boldsymbol{E}$, but for

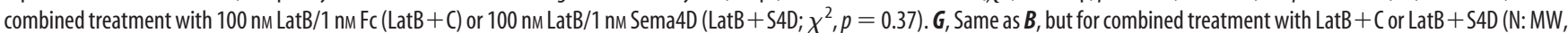
$\left.{ }^{* *} p=0.005 ; \mathrm{L}: \mathrm{MW}, p=0.58 ; \mathrm{S}: \mathrm{MW}, p=0.96 ; \mathrm{D}: \mathrm{MW}, p=0.82 ; \mathrm{I}: \mathrm{MW}, p=0.52\right)$. Data are represented as mean $\pm \mathrm{SEM}$. Data in $\boldsymbol{B}$ are from 21 control axons ( $N=6$ ) and $20 \mathrm{Jasp}$-treated axons $(N=5)$; in $\boldsymbol{C}$ from 18 control axons $(N=5)$ and 20 LatB-treated axons $(N=5)$; and in $\boldsymbol{F}$ and $\boldsymbol{G}$ from 18 LatB + Fc-treated $(N=4)$ and 20 LatB $+S 4 D$-treated axons $(N=5)$.

suggest that LatB and Sema4D treatment act to stabilize a specific, overlapping, subset of inhibitory boutons.

We then wondered whether treatment with the actin depolymerizing drug LatB would be sufficient to induce inhibitory synapse formation, similar to Sema4D signaling (Fig. $3 F$ ). Interestingly, we observed that although LatB induced changes in VGAT area and gephyrin puncta density after $2 \mathrm{~h}$ (Fig. $6 A-F)$, these changes were not coordinated and did not result in an increase in the density of inhibitory synapses (Fig. 6G). The changes in gephyrin and VGAT staining returned to baseline with longer LatB treatment. Together, our data suggest that LatB and Sema4D induce rapid stabilization of the same subgroup of inhibitory boutons, but that only Sema4D signaling leads to coordinated presynaptic and postsynaptic changes resulting in inhibitory synapse formation. This indicates that presynaptic bouton stabilization alone is not enough to induce inhibitory synapse formation, which requires further signaling mechanisms.

Inhibitory bouton stabilization by Sema4D requires MET activation

Our observation that LatB could mimic the Sema4D-induced stabilization of inhibitory boutons points to a possible involvement of MET, as coactivation of MET mediates the actin depolymerization pathway downstream of Sema4D/PlexinB1 signaling (Swiercz et al., 2008). We therefore assessed whether MET activation is necessary for the Sema4D-induced bouton stabilization by making use of the highly specific MET inhibitor PHA-665752 

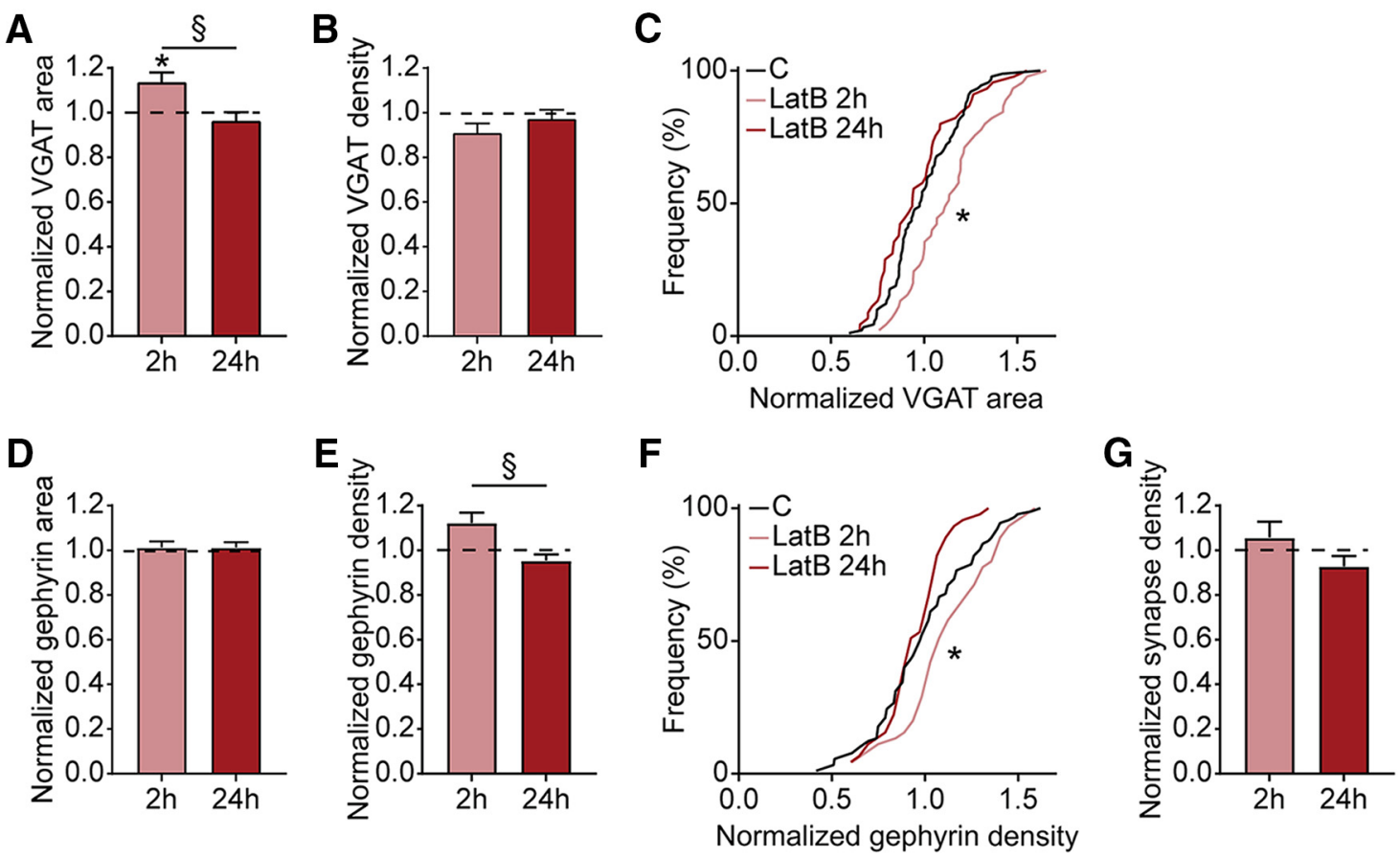

Figure 6. Latrunculin B treatment does not promote inhibitory synapse formation. A, Normalized area of presynaptic VGAT puncta after treatment with $100 \mathrm{~nm}$ LatB for 2 and $24 \mathrm{~h}$. Two-way ANOVA indicated a significant effect of time $(p=0.02$ ) and an interaction between treatment and time ( $\$ p=0.02)$. At $2 \mathrm{~h},{ }^{*} p=0.047$ (Sidak's multiple comparisons test). $\boldsymbol{B}$, Normalized density of VGAT, after treatment with $100 \mathrm{~nm}$ LatB for 2 and $24 \mathrm{~h}$. C, Cumulative distributions of the normalized area of VGAT after treatment with $100 \mathrm{~nm}$ LatB for 2 and $24 \mathrm{~h}$. Black line represents the normalized control values. ${ }^{*} p=0.047$ and $0.33(\mathrm{KS})$ for 2 and $24 \mathrm{~h}$, respectively. $\boldsymbol{D}, \boldsymbol{E}$, Same as in $\boldsymbol{A}$ and $\boldsymbol{B}$, but for the area $(\boldsymbol{D})$ and density $(\boldsymbol{E})$ of postsynaptic gephyrin puncta. $\boldsymbol{E}$, Two-way ANOVA showed a significant effect of time $(p=0.04)$ and interaction between treatment and time $(\$ p=0.04)$. $\boldsymbol{F}$, Same as in $\boldsymbol{C}$, but for normalized gephyrin density. ${ }^{*} p=0.047$ and $0.33(\mathrm{KS})$ for 2 and $24 \mathrm{~h}$, respectively. G, Normalized density of inhibitory synapses. Data are represented as mean \pm SEM. Data from 15 control images $(N=3)$ and 15 LatB images $(N=3)$ per TP. Dashed line represents control values (treatment with DMSO for 2 and $24 \mathrm{~h}$ ).

(PHA; Christensen et al., 2003; Lim and Walikonis, 2008). We first verified that adding PHA alone did not affect bouton dynamics (Fig. $7 A$ ) or spontaneous IPSCs (Fig. $7 B-D$ ), indicating that MET is not very active under baseline conditions in our slices. Next, we treated our slices with Sema4D to induce bouton stabilization and compared bouton dynamics in the presence or absence of PHA (Fig. 7E). Blocking MET with PHA completely abolished the Sema4D-induced increase in the density of stabilizing boutons, while the other bouton subgroups were hardly affected (Fig. $7 F$ ). In fact, blocking MET in combination with Sema4D treatment almost entirely abolished the occurrence of stabilizing boutons in our slices (Fig. $7 G, H$ ). Consistent with the live imaging data, inhibiting MET with PHA also blocked the increase in VGAT staining intensity (Fig. $7 \mathrm{I}, \mathrm{J}$ ) and mIPSC interevent interval (Fig. $7 K$ ) triggered by Sema4D treatment. Together, these data indicate that activation of MET is required for the Sema4D-induced stabilization of inhibitory boutons.

As the actin depolymerization pathway downstream of Sema4D/PlexinB1 signaling via MET is mediated via a reduction of intracellular RhoA activity (Swiercz et al., 2008; Sun et al., 2012), we tested whether stabilization of inhibitory boutons could also be achieved by directly reducing ROCK activity, a well known downstream effector of RhoA (Amano et al., 2010). We found that treatment with the specific ROCK inhibitor Y-27632 (Deguchi et al., 2016) resulted in an increase in the density of stabilizing boutons in our slices (Fig. $7 L$ ), without affecting the other subclasses of boutons. These findings are consistent with an intracellular pathway induced by Sema4D/PlexinB1 signaling via coactivation of MET and reduction of ROCK activity to promote stabilization of inhibitory boutons.

\section{MET is required in presynaptic inhibitory axons}

Our pharmacological experiments do not address whether the Sema4D-induced changes in actin occur in the presynaptic or postsynaptic compartment. MET was reported to be expressed predominantly in axons, where it is present in small puncta in the axonal shaft and small presynaptic terminals (Judson et al., 2009; Eagleson et al., 2013). However, studies on MET have focused on glutamatergic synapses (Tyndall and Walikonis, 2006; Xie et al., 2016) and MET localization in GABAergic axons has never been directly addressed. We made use of an antibody (Qiu et al., 2014) and a nanobody (Heukers et al., 2014) with demonstrated specificity for MET to localize MET in our slices. We first confirmed that MET was present at synapses in primary hippocampal cultures (Fig. 8A,B). In line with previous reports (Tyndall and Walikonis, 2006; Eagleson et al., 2013; Xie et al., 2016), the majority of MET puncta overlapped with excitatory synapses (Fig. $8 A, B)$, but clear association of MET with inhibitory pre-synapses was also observed in these cultures (Fig. $8 B$ ). This was further confirmed with STED microscopy (Fig. $8 C$ ). We then used the MET nanobody and antibody to label MET in our hippocampal slices of GAD65-GFP mice (Fig. 8D). Although there was a quantitative difference presumably reflecting a difference in labeling affinity, both methods clearly showed that a subset of GFPlabeled inhibitory boutons was enriched for MET (Fig. 8E). Comparison between the MET staining pattern with staining for postsynaptic gephyrin (compare Figs. $8 F, 3 E$ ) suggests a presynaptic localization of MET at these inhibitory synapses, as MET puncta were often completely enclosed by the GFP-labeled bouton. These data indicate that MET is present at inhibitory axons 
A

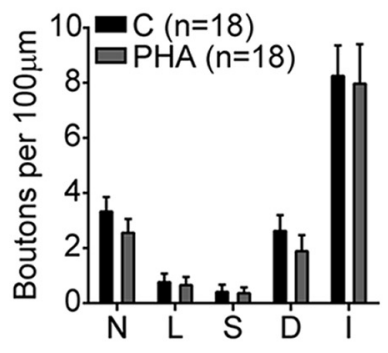

B

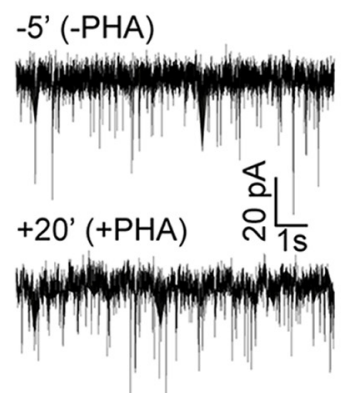

C

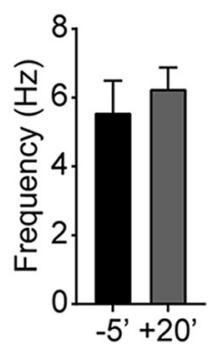

D

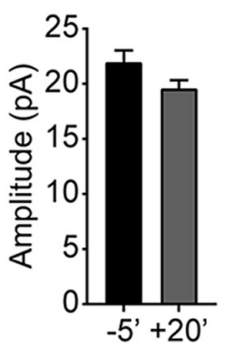

$\mathbf{F}$

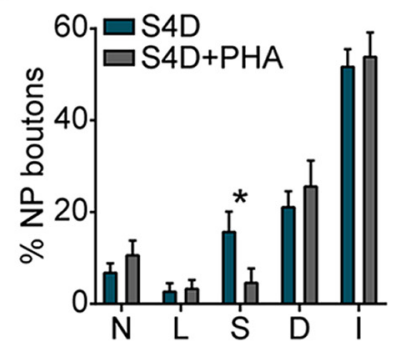

E

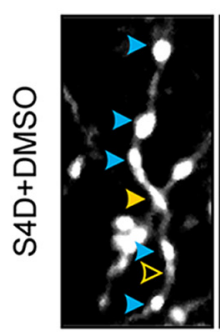

$0^{\prime}$

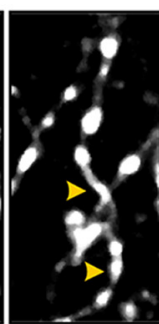

$20^{\prime}$

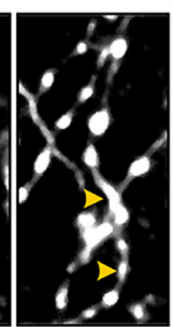

40

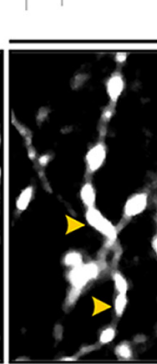

$60^{\prime}$

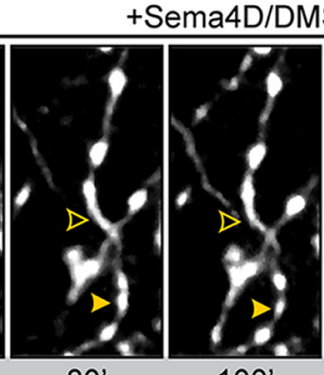

\section{So}
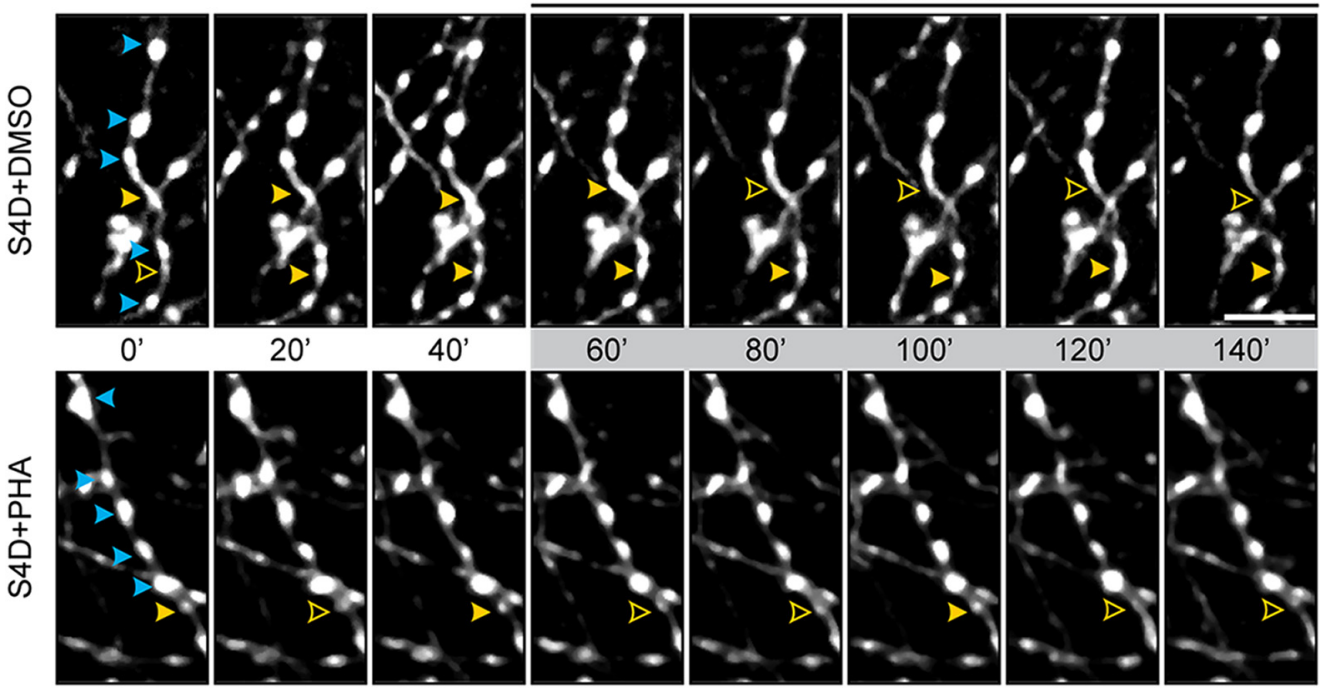

80

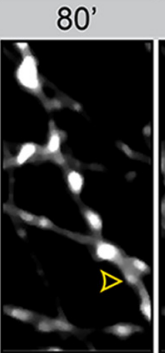

$100^{\prime}$

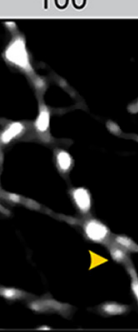

120

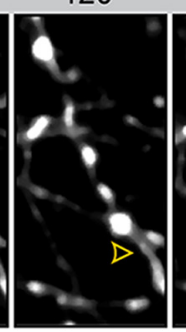

+Sema4D/PHA-665752

H

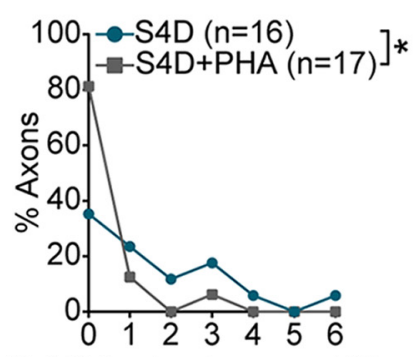

Stabilizing boutons per $100 \mu \mathrm{m}$
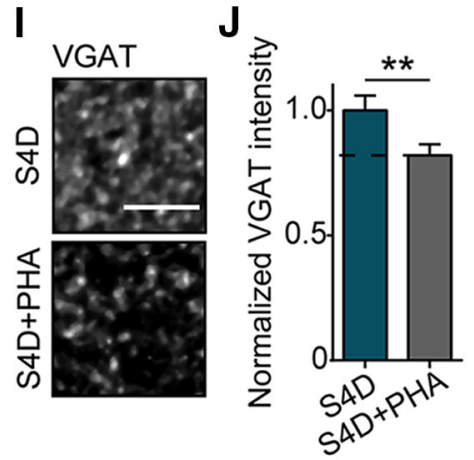

$\mathbf{K}$

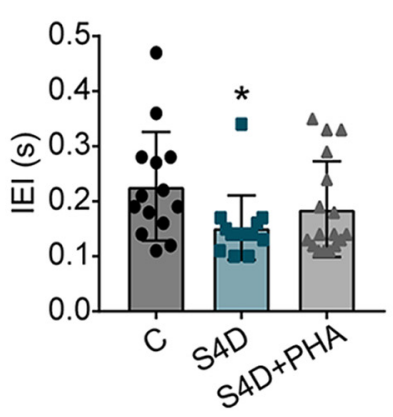

G $\stackrel{\xi}{5}$

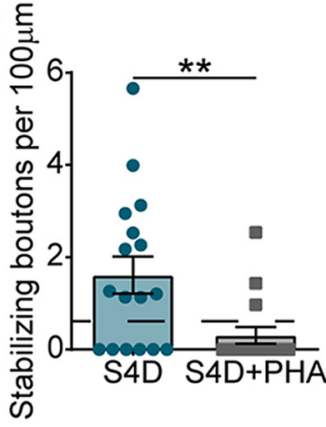

> Persistent bouton

Non-persistent bouton present

$>$ Non-persistent bouton absent

Figure 7. Inhibitory bouton stabilization by Sema4D requires MET activation. $A$, Density of NP boutons in slices treated with DMSO (C) and $1 \mu \mathrm{M}$ PHA-665752 (PHA): N: new (MW, $p=0.28$ ); L: lost (MW, $p=0.77$ ); S: stabilizing (MW, $p=0.98$ ); D: destabilizing (MW, $p=0.24$ ); I: intermittent (MW, $p=0.67)$. B, Representative whole-cell voltage-clamp recordings of spontaneous IPSCS $(s I P S C s)$ from CA1 pyramidal cells in organotypic hippocampal slices before $\left(-5^{\prime}\right)$ and after treatment $\left(+20^{\prime}\right)$ with PHA $(1 \mu \mathrm{M})$. Time in minutes. $\boldsymbol{C}, \boldsymbol{D}$, Mean sIPSC frequency $(\boldsymbol{C})$ and amplitude $(\boldsymbol{D})$ before $\left(-5^{\prime}\right)$ and after $\left(+20^{\prime}\right)$ treatment with PHA. Time in minutes. One-way ANOVA for multiple TPs: $p=0.97(\boldsymbol{C}), p=0.19(\boldsymbol{D})$. E, Time-lapse two-photon images of GAD65-GFP-labeled axons in organotypic hippocampal slices during wash-in (gray box) of combination of $1 \mathrm{~nm}$ Sema4D and DMSO (S4D; top) or combination of $1 \mathrm{~nm}$ Sema4D with $1 \mu \mathrm{M}$ PHA-665752 (S4D + PHA; bottom). 0nly every second image is shown for clarity. Pand NP boutons are indicated as in Figure 2. Images are maximum intensity projections of 15-16z-stacks. Scale bar, $5 \mu \mathrm{m}$. $F$, Fraction of NP boutons in S4Dand S4D + PHA-treated axons: N: MW, $p=0.34 ; \mathrm{L}: \mathrm{MW}, p=0.74 ; \mathrm{S}: \mathrm{MW},{ }^{*} p=0.01 ; \mathrm{D}: \mathrm{MW}, p=0.64 ; \mathrm{I}: \mathrm{MW}, p=0.53$. G, Density of stabilizing boutons in slices treated with S4D or S4D + PHA $\left(M W,{ }^{* *} p=0.006\right)$. Dashed line represents control values. Dots represent individual axons. $\boldsymbol{H}$, Frequency distribution of the stabilizing bouton density in S4D- and S4D + PHA-treated axons $\left(\chi^{2}\right.$, ${ }^{*} p=0.048$ ). I, Representative images of hippocampal slices treated with S4D (top) or S4D+PHA (bottom) for 100', and stained for presynaptic VGAT. Images are average intensity projections of five $z$-stacks. Scale bar, $5 \mu \mathrm{m}$. J, Normalized mean staining intensity for VGAT in S4D- and S4D+PHA-treated slices (MW, $\left.{ }^{* *} p=0.009\right)$. Control value is indicated with dashed line. $\boldsymbol{K}$, Inter-event interval (IEI) of mIPSCs from CA1 pyramidal cells in organotypic slices after treatment with $1 \mathrm{~nm} \mathrm{FC/DMSO} \mathrm{(C),} 1 \mathrm{~nm} \mathrm{S4D/DMSO} \mathrm{(S4D),} \mathrm{or} 1 \mathrm{~nm} \mathrm{S4D/1} \mu \mathrm{m}$ PHA-665752 (S4D + PHA) for 24 h (KW, C vs $S 4 D,{ }^{*} p=0.04 ;$ ( vs PHA, $p=0.19$ ). Dots represent individual cells. L, Fraction of NP boutons in axons treated with MQ (control; C) or $10 \mu \mathrm{M}$ ROCK inhibitor Y-27632: N: MW, $p=0.05 ; \mathrm{L}: \mathrm{MW}, p=$ $0.39 ; \mathrm{S}: \mathrm{MW},{ }^{*} p=0.02 ; \mathrm{D}: \mathrm{MW}, p=0.38 ; \mathrm{I}: \mathrm{MW}, p=0.78$. Data are represented as mean \pm SEM. Data in $A$ are from 18 control axons $(N=4)$ and 18 PHA-treated axons $(N=4) ;$ in $C$ and $D$ from five CA1 pyramidal cells $(N=5)$; in $\boldsymbol{F}-\boldsymbol{H}$ from 17 S4D-treated axons $(N=4)$ and 16 S4D +PHA-treated axons $(N=4)$; in $\boldsymbol{J}$ from 16 images of S4D-treated slices $(N=3)$ and 23 images of S4D+PHA-treated slices $(N=4)$; in $K$ from 14 control cells $(N=5)$, 14S4D-treated cells $(N=7)$ and 17 S4D + PHA-treated cells $(N=6)$; and in $L$ from 21 control axons $(N=5)$ and 22 Y-27632-treated axons $(N=5)$. 
A

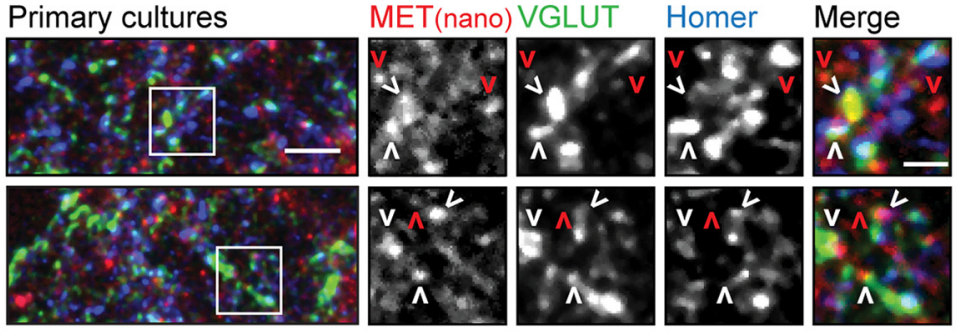

$\gg$ Co-localizing MET > Non-localizing MET

B
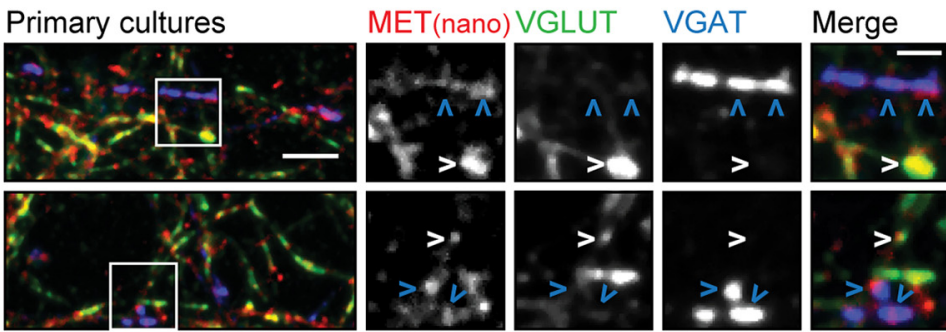

$\gg$ MET co-localizing with VGLUT $>$ MET co-localizing with VGAT

C
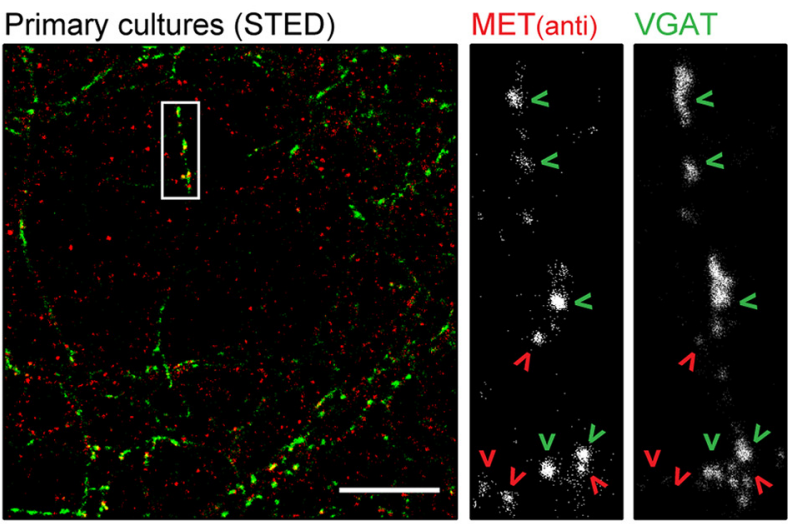

Merge

$>$ MET co-localizing with VGAT > Non-localizing MET

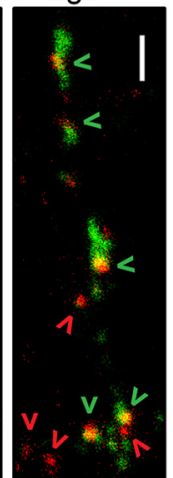

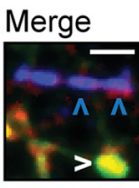

D

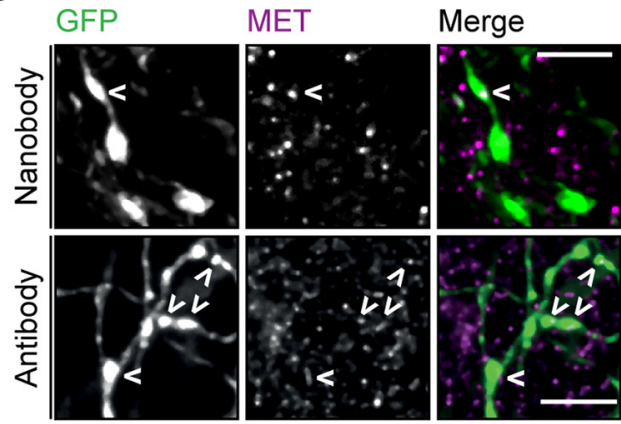

E

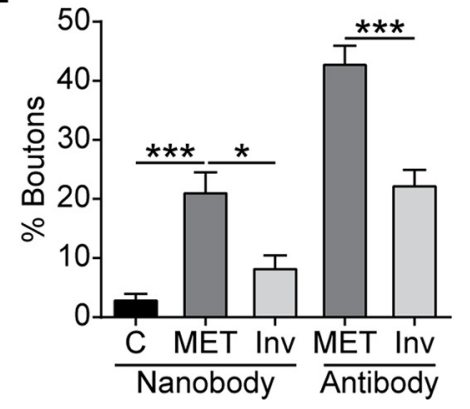

$\mathbf{F}$

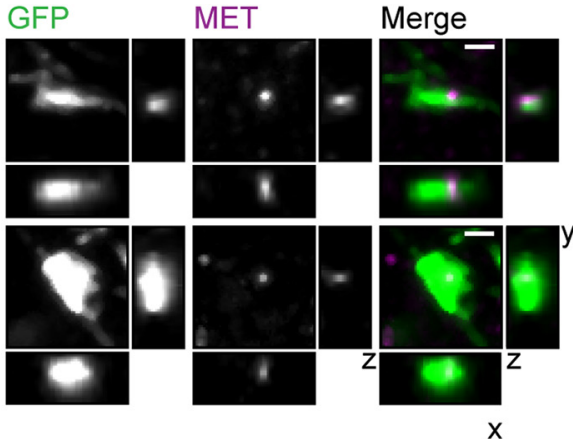

G

$\mathrm{H}$

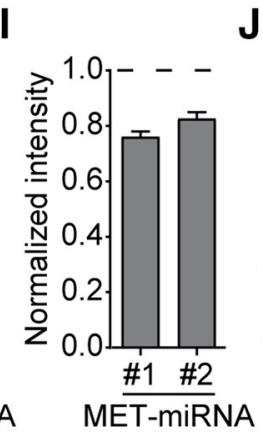

$\mathrm{J}$ 至

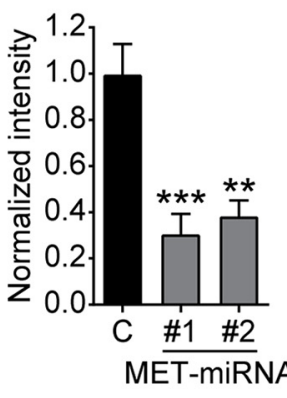

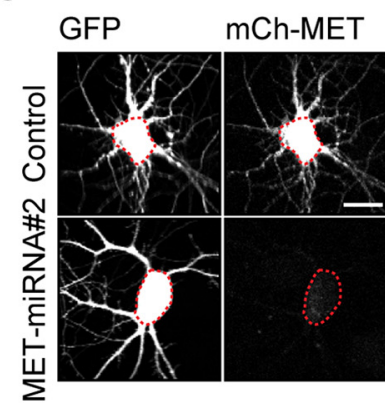

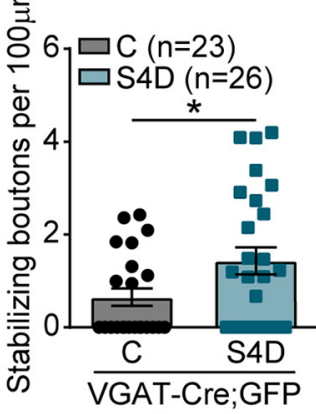

K巨

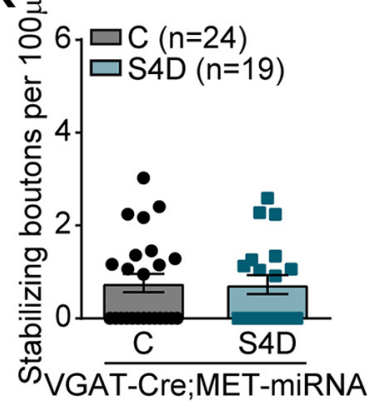

Figure 8. MET is required in presynaptic inhibitory axons. A, Images of primary cultures of hippocampal neurons immunostained with MET nanobody (red) and markers for excitatory synapses: presynaptic vesicular glutamate transporter (VGLUT; green) and postsynaptic Homer (blue). The majority of MET puncta colocalize with one or both markers (white arrows), but some MET puncta do not colocalize (red arrows). Images are maximum intensity projections of 13 stacks. Scale bars: overview, $5 \mu \mathrm{m} ; 200 \mathrm{~m}, 2 \mu \mathrm{m}$. $\boldsymbol{B}$, Same as $\boldsymbol{A}$, but neurons were stained with MET nanobody (red) and markers for excitatory pre-synapses (VGLUT; green) and inhibitory pre-synapses (VGAT; blue). White arrows indicate MET colocalizing with VGLUT and blue arrows indicate MET colocalizing with VGAT. Images are maximum intensity projections of 12 stacks. Scale bars: overview, $5 \mu \mathrm{m} ; z 00 \mathrm{~m}, 2 \mu \mathrm{m}$. C, Representative STED images (single optical sections) of primary cultures of hippocampal neurons immunostained with MET antibody (red) and VGAT (green). Green arrows highlight MET puncta colocalizing with VGAT, while red arrows indicate MET puncta that do not colocalize with VGAT. Scale bars: overview, $10 \mu \mathrm{m} ;$ zoom, $1 \mu \mathrm{m}$. D, Representative images of GFP-labeled inhibitory boutons (green) in hippocampal slices, stained with a nanobody (top) and an antibody (bottom) against MET (magenta). Images are maximum intensity projections of 5-6z-stacks. White arrows indicate MET enrichment in GFP-labeled boutons. Scale bars, $5 \mu \mathrm{m}$. $\boldsymbol{E}$, Fraction of GFP boutons positive for MET. Aspecific staining was determined by anti-myc staining without nanobody (C; black) and random colocalization was determined by inverting the MET channel (Inv; light gray; nanobody-KW, C vs MET: ${ }^{* * *} p=0.0002$, MET vs Inv: ${ }^{*} p=0.04$; antibody-MW: ${ }^{* * *} p<0.0001$ ). $\boldsymbol{F}$, Example of two inhibitory boutons (green) in hippocampal slices showing enrichment in MET (magenta), and the respective xz and yz projections. Images are maximum intensity projections of sixz-stacks. Scale bar, $1 \mu \mathrm{m}$. G, Representative images of primary hippocampal neurons (11 DIV) transfected at 9 DIV with mCherry-MET (mCh-MET) together with GFP (control) or MET-miRNA\#2+GFP (MET-miRNA\#2). Red dashed line highlights the neuronal cell body. Scale bar, $20 \mu \mathrm{m}$. $\boldsymbol{H}$, Normalized intensity of mCherry-MET in primary hippocampal neurons cotransfected with GFP (control) or MET-miRNA constructs (KW: Cvs\#1, ${ }^{* * *} p<0.0001$; (vs\#2, ${ }^{* *} p=0.001$ ).I, Western blot analysis of MET levels in HEK293 cell extracts upon transfection with mCherry-MET and MET-miRNA constructs normalized to control (GFP). (Figure legend continues.) 
and terminals, where it can mediate Sema4D signaling to induce bouton stabilization.

To directly test whether presynaptic MET is required for Sema4D-induced bouton stabilization we reduced MET specifically in presynaptic inhibitory axons using a miRNA-based knock down approach. We first verified that the miRNA construct effectively reduced MET levels in primary hippocampal neurons (Fig. 8G,H) and HEK cells (Fig. 8I). We then injected Cre-dependent lentiviruses in hippocampal slices from VGATCre mice at 1 DIV to infect a few GABAergic cells per slice. We either used a construct with GFP (control), or GFP with miRNA against MET (METmiRNA), and we performed similar twophoton experiments at $>14$ DIV as previously described. We first confirmed that Sema4D promoted bouton stabilization in GFP-positive axons in VGAT-Cre slices (Fig. 8J). As GFP-labeled axons in VGAT-Cre slices comprise all inhibitory axons (compared with only a subset in GAD65-GFP slices), this suggests that many, if not all, inhibitory axons are sensitive to Sema4D signaling. In METmiRNA axons, in which MET levels were reduced for several days, overall bouton dynamics appeared slightly enhanced (fraction of NP boutons in MET-miRNA axons was $20.9 \pm 1.6 \%$ compared with $15.5 \pm 1.7 \%$ in control GFP axons; $p=$ $0.04)$. In contrast to GFP-positive axons, Sema4D was not able to increase the density of stabilizing boutons along METmiRNA axons (Fig. 8K). These data demonstrate that Sema4D-induced inhibitory bouton stabilization requires the coactivation of presynaptic MET in inhibitory axons.

\section{Sema4D-induced bouton stabilization relies on network activity}

Finally, we asked whether Sema4D-induced stabilization of inhibitory boutons depends on the level of neuronal activity. As previously reported (Schuemann et al., 2013), blocking activity by bath application of TTX mildly decreased overall bouton dynamics in our slices (data not shown). We found that in the presence of TTX, Sema4D treatment no longer induced stabilization of inhibitory boutons, and that Sema4D treatment even led to a reduction in

\section{$\leftarrow$}

(Figure legend continued.) Dashed line represents control.J, Density of stabilizing boutons in GFP-positive axons treated with $1 \mathrm{~nm} \mathrm{Fc} \mathrm{(C)} \mathrm{and} 1 \mathrm{~nm}$ Sema4D-Fc (S4D) in VGAT-Cre slices (MW, ${ }^{*} p=0.05$ ). $\boldsymbol{K}$, Same as in $\boldsymbol{J}$, but in MET-miRNA-expressing axons (MW, $p=0.88$ ). Data are represented as mean \pm SEM. Data in $\boldsymbol{E}$ are from 10 control images $(N=2), 12$ images in MET and inverted group $(N=3)$ for the nanobody staining and 15 images in MET and inverted group $(N=3)$ for the antibody staining; in $\boldsymbol{H}$ from 45 control cells $(N=3), 40$ MET-miRNA\#1 cells $(N=2)$ and 43 MET-miRNA\#2 cells $(N=3)$; in I from 2 independent Western blot experiments; in $J$ from 23 control axons $(N=6)$ and 26S4D-treated axons $(N=7)$; and in $\boldsymbol{K}$ from 24 control axons $(N=6)$ and 19 S4D-treated axons $(N=5)$. Dots in $J, K$ represent individual axons.
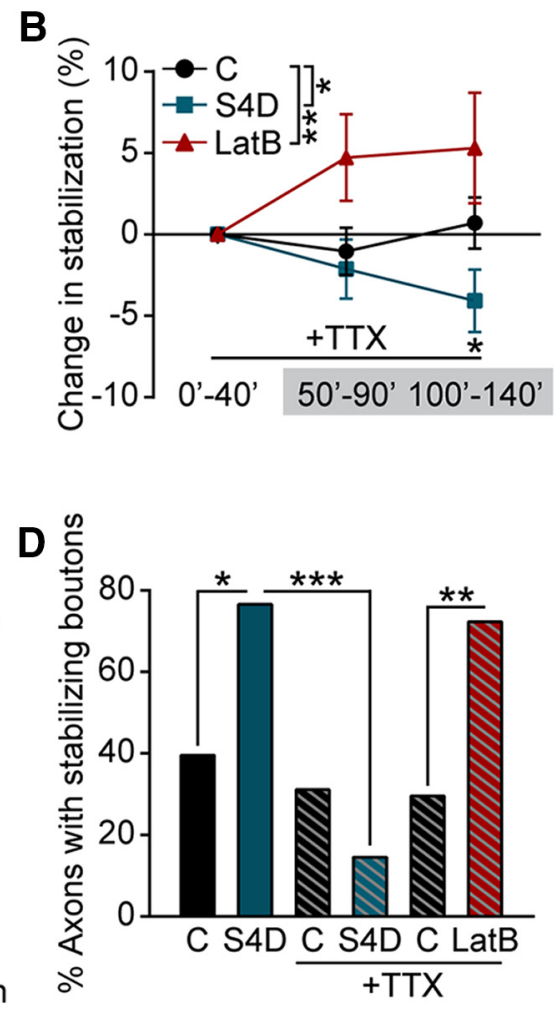

Figure 9. Sema4D-induced bouton stabilization is activity-dependent. $\boldsymbol{A}$, Density of stabilizing boutons in axons treated with 1 S4D, or LatB in the presence of $0.5 \mu \mathrm{m}$ TTX, determined by the change (compared with baseline) in density of boutons that

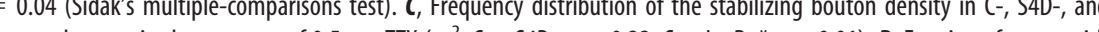
between treatment and its own control.

bouton stabilization compared with control (Fig. 9A,B). Whereas under control conditions Sema4D treatment increased the fraction of axons that displayed stabilizing boutons, it led to a decrease in the presence of TTX (Fig. 9C,D). Interestingly, bouton stabilization by LatB was not affected by TTX (Fig. 9A-D), indicating that activitydependent changes in the actin cytoskeleton cannot explain this result. These findings suggest that the activity-dependent sensitivity to Sema4D is regulated upstream of the changes induced at the presynaptic actin cytoskeleton.

\section{Discussion}

Research on synapse formation is traditionally dominated by excitatory synapses and has been focused on the postsynaptic side. Inhibitory synapses are formed in a fundamentally different manner, with a more prominent role for the presynaptic axon (Wierenga et al., 2008). Our live imaging experiments provide unique insight into the dynamics of inhibitory synapse formation in brain slices, which remain undetected with methods using stationary comparisons before and after treatment. We used Sema4D-induced inhibitory synapse formation as a model system to study the molecular events happening during presynaptic bouton formation. Rather than being specific for a certain subtype of inhibitory cell, our observations indicate that Sema4D 
acts at a specific molecular step during inhibitory synapse formation. Our current data put the presynaptic inhibitory axon in the spotlight and highlights the importance of understanding the precise presynaptic changes and signaling events that occur during the formation of new inhibitory synapses.

We demonstrate that the primary action of Sema4D is on the presynaptic side. During the formation of inhibitory synapses, synaptic changes generally occur in a pre-before-post sequence (Wierenga et al., 2008; Dobie and Craig, 2011), but it was recently reported that the formation of inhibitory synapses can also be induced by postsynaptic gephyrin clustering (Flores et al., 2015). A previous observation of rapid formation of new gephyrin clusters after Sema4D treatment in primary cultures (Kuzirian et al., 2013) suggested that Sema4D may promote inhibitory synapse formation via postsynaptic gephyrin. However, our data clearly argue against a triggering mechanism of Sema4D via postsynaptic gephyrin and indicates that the primary action of Sema4D is at the presynaptic bouton. We found that Sema4D signaling does not induce de novo inhibitory synapse formation, but it specifically stabilizes NP boutons within tens of minutes, without affecting bouton disassembly. Initial presynaptic bouton stabilization is followed by a slower postsynaptic recruitment of gephyrin and the entire synapse maturation process takes several (up to 24) hours. Our results highlight the importance of the presynaptic changes that occur during inhibitory synapse formation.

Our observations that longer Sema4D treatment does not further enhance bouton stabilization and that LatB and Sema4D stabilize an overlapping bouton population suggests that only a specific set of boutons is responsive to Sema4D. This indicates that Sema4D acts at a specific, early step during the synapse formation process and inhibitory boutons which are more mature or too immature are irresponsive to Sema4D. Bouton stabilization and gephyrin recruitment was also induced by LatB, but in an uncoordinated manner, such that LatB failed to induce new inhibitory synapses after bouton stabilization (Fig. 6). This suggests that Sema4D signaling coordinates presynaptic and postsynaptic changes, and that independent signaling pathways exist for presynaptic and postsynaptic changes during inhibitory synapse formation. This is in line with two recent studies, which show that impairing clustering of postsynaptic gephyrin does not affect presynaptic bouton stabilization (Yamasaki et al., 2017; Wu et al., 2018). Gephyrin clustering at synapses is a complex process, which is regulated via phosphorylation-dependent interactions with multiple postsynaptic proteins and $\mathrm{GABA}_{\mathrm{A}}$ receptors $(\mathrm{Pe}-$ trini et al., 2014; Tyagarajan and Fritschy, 2014; Flores et al., 2015; Oh et al., 2016; Yamasaki et al., 2017; Wu et al., 2018). It is currently not known how presynaptic bouton stabilization triggers subsequent recruitment of postsynaptic proteins, but this may involve the recruitment of presynaptic organizers such as neurexins (Fu and Huang, 2010; Siddiqui and Craig, 2011; Neupert et al., 2015).

Stabilization of inhibitory boutons involves specific actin remodeling via actin depolymerization downstream of Sema4D signaling. This might seem counterintuitive at first sight, but a similar rapid actin depolymerization was implied in building specific actin structures during spine growth (Bosch et al., 2014; Meyer et al., 2014) and in the formation of immunological synapses (Ritter et al., 2015; de la Roche et al., 2016). Actin remodeling likely involves a qualitative switch in the combined action of many actin-regulating proteins (Lomakin et al., 2015; Rotty et al., 2015; Suarez et al., 2015). Our data suggest that the actin cytoskeleton at stabilizing boutons is different from other compartments and specifically sensitive to LatB. Recent nanoscopy studies have revealed several actin-based structures in axons (Xu et al., 2013; Ganguly et al., 2015; Leterrier et al., 2017), but the precise actin structure in presynaptic boutons remains unresolved. It is currently not known which actin-regulating factors are involved in presynaptic bouton stabilization, but promising candidates include cortactin (Alicea et al., 2017), cofilin (Bosch et al., 2014; Piccioli and Littleton, 2014), and Mical (Orr et al., 2017).

Our experiments uncovered an unexpected role for the receptor tyrosine kinase MET in inhibitory synapses. Human imaging and genetic studies have identified mutations in the MET gene as a risk factor for autism spectrum disorder (ASD; Peng et al., 2013), but the exact role of MET in ASD is not yet understood (Eagleson et al., 2017). Previous studies in neurons have implicated MET in regulating postsynaptic strength in excitatory neurons (Qiu et al., 2014; Lo et al., 2016), excitatory synapse formation (Xie et al., 2016), and interneuron migration (Martins et al., 2011). We found that reducing MET levels specifically in inhibitory axons blocked Sema4D-induced inhibitory bouton stabilization, suggesting that presynaptic MET acts as a coreceptor with PlexinB1 (or other family members; McDermott et al., 2018) to mediate actin remodeling in the presynaptic bouton. This novel role for MET in inhibitory axons could shed new light on its role in neurodevelopmental diseases such as ASD.

Finally, we found that the sensitivity to Sema4D signaling is regulated by activity. Several members of the Semaphorin and Plexin family have been shown to be regulated by neuronal activity (Orr et al., 2017; Wang et al., 2017). Our experiments suggest that intracellular actin is not changed, but that the sensitivity for Sema4D is altered in the axon, possibly by activity-dependent regulation of surface expression or complex formation of receptors. It will be important to further address the activitydependent regulation of the Sema4D signaling pathway, including PlexinB and MET receptors, in future studies. Changes in inhibitory synapses play an important role in the rewiring of neural circuits during development and in response to behavioral demands in adulthood (Keck et al., 2011; Chen et al., 2015; Froemke, 2015) and defects in inhibitory synapses are associated with many neurodevelopmental diseases (Hensch, 2004; Marín, 2012). Our data emphasize that inhibitory synapses are not plugand-play devices, but that the activity-dependent formation of new inhibitory synapses requires a complex series of molecular events. A better understanding of the signaling pathways that regulate these events will be crucial to understand circuit adaptation processes during learning and in brain diseases.

\section{References}

Alicea D, Perez M, Maldonado C, Dominicci-Cotto C, Marie B (2017) Cortactin is a regulator of activity-dependent synaptic plasticity controlled by wingless. J Neurosci 37:2203-2215.

Amano M, Nakayama M, Kaibuchi K (2010) Rho-kinase/ROCK: a key regulator of the cytoskeleton and cell polarity. Cytoskeleton 67:545-554.

Bosch M, Castro J, Saneyoshi T, Matsuno H, Sur M, Hayashi Y (2014) Structural and molecular remodeling of dendritic spine substructures during long-term potentiation. Neuron 82:444-459.

Bury LA, Sabo SL (2016) Building a terminal: mechanisms of presynaptic development in the CNS. Neuroscientist 22:372-391.

Cagnoni G, Tamagnone L (2014) Semaphorin receptors meet receptor tyrosine kinases on the way of tumor progression. Oncogene 33:4795-4802.

Caroni P, Donato F, Muller D (2012) Structural plasticity upon learning: regulation and functions. Nat Rev Neurosci 13:478-490.

Cellot G, Cherubini E (2014) GABAergic signaling as therapeutic target for autism spectrum disorders. Front Pediatr 2:70.

Chen SX, Kim AN, Peters AJ, Komiyama T (2015) Subtype-specific plasticity of inhibitory circuits in motor cortex during motor learning. Nat Neurosci 18:1109-1115. 
Christensen JG, Schreck R, Burrows J, Kuruganti P, Chan E, Le P, Chen J, Wang X, Ruslim L, Blake R, Lipson KE, Ramphal J, Do S, Cui JJ, Cherrington JM, Mendel DB (2003) A selective small molecule inhibitor of c-met kinase inhibits c-met-dependent phenotypes in vitro and exhibits cytoreductive antitumor activity in vivo. Cancer Res 63:7345-7355.

de la Roche M, Asano Y, Griffiths GM (2016) Origins of the cytolytic synapse. Nat Rev Immunol 16:421-432.

Deguchi Y, Harada M, Shinohara R, Lazarus M, Cherasse Y, Urade Y, Yamada D, Sekiguchi M, Watanabe D, Furuyashiki T, Narumiya S (2016) mDia and ROCK mediate actin-dependent presynaptic remodeling regulating synaptic efficacy and anxiety. Cell Rep 17:2405-2417.

Dobie FA, Craig AM (2011) Inhibitory synapse dynamics: coordinated presynaptic and postsynaptic mobility and the major contribution of recycled vesicles to new synapse formation. J Neurosci 31:10481-10493.

Du G, Yonekubo J, Zeng Y, Osisami M, Frohman MA (2006) Design of expression vectors for RNA interference based on miRNAs and RNA splicing. FEBS J 273:5421-5427.

Eagleson KL, Milner TA, Xie Z, Levitt P (2013) Synaptic and extrasynaptic location of the receptor tyrosine kinase met during postnatal development in the mouse neocortex and hippocampus. J Comp Neurol 521: 3241-3259.

Eagleson KL, Xie Z, Levitt P (2017) The pleiotropic MET receptor network: circuit development and the neural-medical interface of autism. Biol Psychiatry 81:424-433.

Esteves da Silva M, Adrian M, Schätzle P, Lipka J, Watanabe T, Cho S, Futai K, Wierenga CJ, Kapitein LC, Hoogenraad CC (2015) Positioning of AMPA receptor-containing endosomes regulates synapse architecture. Cell Rep 13:933-943.

Flores CE, Nikonenko I, Mendez P, Fritschy JM, Tyagarajan SK, Muller D (2015) Activity-dependent inhibitory synapse remodeling through gephyrin phosphorylation. Proc Natl Acad Sci U S A 112:E65-E72.

Frias CP, Wierenga CJ (2013) Activity-dependent adaptations in inhibitory axons. Front Cell Neurosci 7:219.

Froemke RC (2015) Plasticity of cortical excitatory-inhibitory balance. Annu Rev Neurosci 38:195-219.

Fu Y, Huang ZJ (2010) Differential dynamics and activity-dependent regulation of $\alpha$ - and $\beta$-neurexins at developing GABAergic synapses. Proc Natl Acad Sci U S A 107:22699-22704.

Fu Y, Wu X, Lu J, Huang ZJ (2012) Presynaptic GABAB receptor regulates activity-dependent maturation and patterning of inhibitory synapses through dynamic allocation of synaptic vesicles. Front Cell Neurosci 6:57.

Ganguly A, Tang Y, Wang L, Ladt K, Loi J, Dargent B, Leterrier C, Roy S (2015) A dynamic formin-dependent deep F-actin network in axons. J Cell Biol 210:401-417.

Hensch TK (2004) Critical period regulation. Annu Rev Neurosci 27:549579.

Hensch TK (2005) Critical period plasticity in local cortical circuits. Nat Rev Neurosci 6:877-888.

Heukers R, Altintas I, Raghoenath S, De Zan E, Pepermans R, Roovers RC, Haselberg R, Hennink WE, Schiffelers RM, Kok RJ, van Bergen en Henegouwen PMP (2014) Targeting hepatocyte growth factor receptor (MET) positive tumor cells using internalizing nanobody-decorated albumin nanoparticles. Biomaterials 35:601-610.

Honkura N, Matsuzaki M, Noguchi J, Ellis-Davies GC, Kasai H (2008) The subspine organization of actin fibers regulates the structure and plasticity of dendritic spines. Neuron 57:719-729.

Isaacson JS, Scanziani M (2011) How inhibition shapes cortical activity. Neuron 72:231-243.

Judson MC, Bergman MY, Campbell DB, Eagleson KL, Levitt P (2009) Dynamic gene and protein expression patterns of the autism-associated met receptor tyrosine kinase in the developing mouse forebrain. J Comp Neurol 513:511-531.

Keck T, Scheuss V, Jacobsen RI, Wierenga CJ, Eysel UT, Bonhoeffer T, Hübener M (2011) Loss of sensory input causes rapid structural changes of inhibitory neurons in adult mouse visual cortex. Neuron 71:869-882.

Kolodkin AL, Matthes DJ, Goodman CS (1993) The semaphorin genes encode a family of transmembrane and secreted growth cone guidance molecules. Cell 75:1389-1399.

Krueger-Burg D, Papadopoulos T, Brose N (2017) Organizers of inhibitory synapses come of age. Curr Opin Neurobiol 45:66-77.

Kuriu T, Yanagawa Y, Konishi S (2012) Activity-dependent coordinated mobility of hippocampal inhibitory synapses visualized with presynaptic and postsynaptic tagged-molecular markers. Mol Cell Neurosci 49:184195.

Kuzirian MS, Moore AR, Staudenmaier EK, Friedel RH, Paradis S (2013) The class 4 semaphorin Sema4D promotes the rapid assembly of GABAergic synapses in rodent hippocampus. J Neurosci 33:8961-8973.

Leterrier C, Dubey P, Roy S (2017) The nano-architecture of the axonal cytoskeleton. Nat Rev Neurosci 18:713-726.

Lim CS, Walikonis RS (2008) Hepatocyte growth factor and c-met promote dendritic maturation during hippocampal neuron differentiation via the akt pathway. Cell Signal 20:825-835.

Lo FS, Erzurumlu RS, Powell EM (2016) Insulin-independent GABAA receptor-mediated response in the barrel cortex of mice with impaired met activity. J Neurosci 36:3691-3697.

Lomakin AJ, Lee KC, Han SJ, Bui DA, Davidson M, Mogilner A, Danuser G (2015) Competition for actin between two distinct F-actin networks defines a bistable switch for cell polarization. Nat Cell Biol 17:1435-1445.

López-Bendito G, Sturgess K, Erdélyi F, Szabó G, Molnár Z, Paulsen O (2004) Preferential origin and layer destination of GAD65-GFP cortical interneurons. Cereb Cortex 14:1122-1133.

Lu W, Bromley-Coolidge S, Li J (2017) Regulation of GABAergic synapse development by postsynaptic membrane proteins. Brain Res Bull 129: $30-42$.

Marín O (2012) Interneuron dysfunction in psychiatric disorders. Nat Rev Neurosci 13:107-120.

Martins GJ, Shahrokh M, Powell EM (2011) Genetic disruption of met signaling impairs GABAergic striatal development and cognition. Neuroscience 176:199-209.

McDermott JE, Goldblatt D, Paradis S (2018) Class 4 semaphorins and plexin-B receptors regulate GABAergic and glutamatergic synapse development in the mammalian hippocampus. Mol Cell Neurosci 92:50-66.

Meyer D, Bonhoeffer T, Scheuss V (2014) Balance and stability of synaptic structures during synaptic plasticity. Neuron 82:430-443.

Müllner FE, Wierenga CJ, Bonhoeffer T (2015) Precision of inhibition: dendritic inhibition by individual GABAergic synapses on hippocampal pyramidal cells is confined in space and time. Neuron 87:576-589.

Nelson SB, Valakh V (2015) Excitatory/inhibitory balance and circuit homeostasis in autism spectrum disorders. Neuron 87:684-698.

Neupert C, Schneider R, Klatt O, Reissner C, Repetto D, Biermann B, Niesmann K, Missler M, Heine M (2015) Regulated dynamic trafficking of neurexins inside and outside of synaptic terminals. J Neurosci 35:1362913647.

Oh WC, Lutzu S, Castillo PE, Kwon HB (2016) De novo synaptogenesis induced by GABA in the developing mouse cortex. Science 353:10371040

Orr BO, Fetter RD, Davis GW (2017) Retrograde semaphorin-plexin signalling drives homeostatic synaptic plasticity. Nature 550:109-113.

Paradis S, Harrar DB, Lin Y, Koon AC, Hauser JL, Griffith EC, Zhu L, Brass LF, Chen C, Greenberg ME (2007) An RNAi-based approach identifies molecules required for glutamatergic and GABAergic synapse development. Neuron 53:217-232.

Pasterkamp RJ (2012) Getting neural circuits into shape with semaphorins. Nat Rev Neurosci 13:605-618.

Peng Y, Huentelman M, Smith C, Qiu S (2013) MET receptor tyrosine kinase as an autism genetic risk factor. Int Rev Neurobiol 113:135-165.

Petrini EM, Ravasenga T, Hausrat TJ, Iurilli G, Olcese U, Racine V, Sibarita JB, Jacob TC, Moss SJ, Benfenati F, Medini P, Kneussel M, Barberis A (2014) Synaptic recruitment of gephyrin regulates surface $\mathrm{GABA}_{\mathrm{A}}$ receptor dynamics for the expression of inhibitory LTP. Nat Commun 5:3921.

Piccioli ZD, Littleton JT (2014) Retrograde BMP signaling modulates rapid activity-dependent synaptic growth via presynaptic LIM kinase regulation of cofilin. J Neurosci 34:4371-4381.

Qiu S, Lu Z, Levitt P (2014) MET receptor tyrosine kinase controls dendritic complexity, spine morphogenesis, and glutamatergic synapse maturation in the hippocampus. J Neurosci 34:16166-16179.

Raissi AJ, Staudenmaier EK, David S, Hu L, Paradis S (2013) Sema4D localizes to synapses and regulates GABAergic synapse development as a membrane-bound molecule in the mammalian hippocampus. Mol Cell Neurosci 57:23-32.

Rex CS, Chen LY, Sharma A, Liu J, Babayan AH, Gall CM, Lynch G (2009) Different rho GTPase-dependent signaling pathways initiate sequential steps in the consolidation of long-term potentiation. J Cell Biol 186: 85-97. 
Ritter AT, Asano Y, Stinchcombe JC, Dieckmann NM, Chen BC, GawdenBone C, van Engelenburg S, Legant W, Gao L, Davidson MW, Betzig E, Lippincott-Schwartz J, Griffiths GM (2015) Actin depletion initiates events leading to granule secretion at the immunological synapse. Immunity 42:864-876.

Rotty JD, Wu C, Haynes EM, Suarez C, Winkelman JD, Johnson HE, Haugh JM, Kovar DR, Bear JE (2015) Profilin-1 serves as a gatekeeper for actin assembly by Arp2/3-dependent and -independent pathways. Dev Cell 32:54-67.

Schuemann A, Klawiter A, Bonhoeffer T, Wierenga CJ (2013) Structural plasticity of GABAergic axons is regulated by network activity and $\mathrm{GABA}_{\mathrm{A}}$ receptor activation. Front Neural Circuits 7:113.

Siddiqui TJ, Craig AM (2011) Synaptic organizing complexes. Curr Opin Neurobiol 21:132-143.

Sprekeler H (2017) Functional consequences of inhibitory plasticity: homeostasis, the excitation-inhibition balance and beyond. Curr Opin Neurobiol 43:198-203.

Staras K (2007) Share and share alike: trading of presynaptic elements between central synapses. Trends Neurosci 30:292-298.

Suarez C, Carroll RT, Burke TA, Christensen JR, Bestul AJ, Sees JA, James ML, Sirotkin V, Kovar DR (2015) Profilin regulates F-actin network homeostasis by favoring formin over Arp2/3 complex. Dev Cell 32:43-53.

Sun T, Krishnan R, Swiercz JM (2012) Grb2 mediates semaphorin-4Ddependent RhoA inactivation. J Cell Sci 125:3557-3567.

Swiercz JM, Worzfeld T, Offermanns S (2008) ErbB-2 and met reciprocally regulate cellular signaling via plexin-B1. J Biol Chem 283:1893-1901.

Tyagarajan SK, Fritschy JM (2014) Gephyrin: a master regulator of neuronal function? Nat Rev Neurosci 15:141-156.

Tyndall SJ, Walikonis RS (2006) The receptor tyrosine kinase met and its ligand hepatocyte growth factor are clustered at excitatory synapses and can enhance clustering of synaptic proteins. Cell Cycle 5:1560-1568.

Villa KL, Berry KP, Subramanian J, Cha JW, Oh WC, Kwon HB, Kubota Y, So PT, Nedivi E (2016) Inhibitory synapses are repeatedly assembled and removed at persistent sites in vivo. Neuron 89:756-769.

Wang Q, Chiu SL, Koropouli E, Hong I, Mitchell S, Easwaran TP, Hamilton NR, Gustina AS, Zhu Q, Ginty DD, Huganir RL, Kolodkin AL (2017) Neuropilin-2/PlexinA3 receptors associate with GluA1 and mediate Sema3F-dependent homeostatic scaling in cortical neurons. Neuron 96:1084-1098.e7.

Wierenga CJ, Becker N, Bonhoeffer T (2008) GABAergic synapses are formed without the involvement of dendritic protrusions. Nat Neurosci 11:1044-1052.

Wierenga CJ, Müllner FE, Rinke I, Keck T, Stein V, Bonhoeffer T (2010) Molecular and electrophysiological characterization of GFP-expressing CA1 interneurons in GAD65-GFP mice. PLoS One 5:e15915.

Wu M, Tian HL, Liu X, Lai JHC, Du S, Xia J (2018) Impairment of inhibitory synapse formation and motor behavior in mice lacking the NL2 binding partner LHFPL4/GARLH4. Cell Rep 23:1691-1705.

Xie Z, Eagleson KL, Wu HH, Levitt P (2016) Hepatocyte growth factor modulates MET receptor tyrosine kinase and $\beta$-catenin functional interactions to enhance synapse formation. eNeuro 3:ENEURO.0074-16. 2016.

Xu K, Zhong G, Zhuang X (2013) Actin, spectrin, and associated proteins form a periodic cytoskeletal structure in axons. Science 339:452-456.

Yamasaki T, Hoyos-Ramirez E, Martenson JS, Morimoto-Tomita M, Tomita S (2017) GARLH family proteins stabilize $\mathrm{GABA}_{\mathrm{A}}$ receptors at synapses. Neuron 93:1138-1152.e6.

Zhou Y, Gunput RA, Pasterkamp RJ (2008) Semaphorin signaling: progress made and promises ahead. Trends Biochem Sci 33:161-170. 